\title{
Un-Reduction of Systems of Second-Order Ordinary Differential Equations
}

\author{
Eduardo GARCÍA-TORAÑO ANDRÉS ${ }^{\dagger}$ and Tom MESTDAG ${ }^{\ddagger}$ \\ † Departamento de Matemática, Universidad Nacional del Sur, CONICET, \\ Av. Alem 1253, 8000 Bahía Blanca, Argentina \\ E-mail: egtoranoandres@gmail.com \\ $¥$ Department of Mathematics and Computer Science, University of Antwerp, \\ Middelheimlaan 1, B-2020 Antwerpen, Belgium \\ E-mail: mestdagtom@gmail.com
}

Received August 12, 2016, in final form November 29, 2016; Published online December 07, 2016 http://dx.doi.org/10.3842/SIGMA.2016.115

\begin{abstract}
In this paper we consider an alternative approach to "un-reduction". This is the process where one associates to a Lagrangian system on a manifold a dynamical system on a principal bundle over that manifold, in such a way that solutions project. We show that, when written in terms of second-order ordinary differential equations (SODEs), one may associate to the first system a (what we have called) "primary un-reduced SODE", and we explain how all other un-reduced SODEs relate to it. We give examples that show that the considered procedure exceeds the realm of Lagrangian systems and that relate our results to those in the literature.
\end{abstract}

Key words: reduction; symmetry; principal connection; second-order ordinary differential equations; Lagrangian system

2010 Mathematics Subject Classification: 34A26; 37J15; 70H33; $70 \mathrm{G} 65$

\section{Introduction}

One of the much-discussed aspects of Lagrangian systems with a symmetry group is their reduction to the so-called Lagrange-Poincaré equations [6] (but see also [9] for an approach using Lie algebroids, or [13] for an approach that is relevant for this paper). The idea is that one may cancel out the symmetry group, and, once a principal connection has been invoked, arrive at two sets of coupled equations on a quotient manifold, the so-called horizontal and vertical equations. The horizontal equation looks again a bit like a Lagrangian equation (for a reduced-type Lagrangian function), but it has extra non-conservative force terms and, equally important, it is in general not decoupled from the vertical equation.

In recent years, there has been some interest in so-called un-reduction $[1,2,5,7]$. The motivation behind this paper is related to the un-reduction theorem (Theorem 5.1) of the paper [5]. Given a Lagrangian $\ell$ on a quotient manifold $\bar{M}=M / G$ and a principal connection $\omega$ on $\pi: M \rightarrow M / G$, the theorem tells one how to associate a "Lagrangian system with extra non-conservative forces" on $M$ which has the property that its solutions project to those of the Euler-Lagrange equations of $\ell$. The methodology and reasoning of [5] is almost entirely based on Lagrange-Poincaré reduction, and on the fact that one may choose the external forces in such a way that the inconvenient curvature terms which appear in the horizontal Lagrange-Poincaré equation vanish. In our opinion, it may be more advantageous to think of this problem outside of the Lagrange-Poincaré framework. The Lagrange-Poincaré equations give essentially a dynamical system on the manifold $(T M) / G$ (a Lie algebroid), while the Euler-Lagrange equations 
of $\ell$ are defined on $T(M / G)$ (a tangent bundle). One of the purposes of this paper is to show that for a comparison of dynamics one may remain in the category of tangent bundles.

The Euler-Lagrange equations of $\ell$ are but a particular example of a SODE, a system of second-order ordinary differential equations. The un-reduced equations one finds in [5] are no longer Euler-Lagrange equations, since the presence of extra non-conservative forces breaks the variational character of these equations, but they are still a SODE. We will show that the unreduction process is very natural in the context of SODEs, and that one may identify a (what we call) "primary un-reduced SODE", to which all other un-reduced SODEs easily relate. There is, in our setting, no need to invoke Lagrange-Poincaré equations or external forces. The issues related to what is called "coupling distortion" and "curvature distortion" in [5] are, in our opinion, side-effects from relying on a Lagrange-Poincaré-based approach to un-reduction (as opposed to a SODE-based approach).

In Section 3 we identify the two lifted principal connections that lie at the basis of our un-reduction. After the definition of the primary un-reduced SODE in Section 4 we state a proposition about all other SODEs whose base integral curves project on those of the given SODE. The canonical connection of a Lie group gives, in Section 5, a natural environment where all the introduced concepts can be clarified. Since it is not always variational (i.e., its geodesics are not always solutions of some Euler-Lagrange equations) it shows that our discussion is a meaningful generalization of the one in [5]. In Section 6 we specify to the case of a Lagrangian SODE, and we discuss the example of Wong's equations and the effect of curvature distortion in Section 7. In Section 8 we say a few words about a second un-reduction procedure. We end the paper with some possible lines of future research.

The advantage of un-reduction remains unaltered in our setting, as it is explained in [5]: If one knows that solutions of a second-order system on $\bar{M}$ are but the projection of those of a system on $M$, one may compute these solutions by making use of any coordinates on $M$, not necessarily those adapted to the bundle structure of $\pi$. This may be beneficial when, e.g., the equations on $M$ are more convenient to deal with numerically, which is precisely one of the main motivations to study un-reduction. We refer the interested reader to [5] and references therein for a more detailed discussion.

\section{Preliminaries}

Consider a manifold $M$ and its tangent bundle $\tau_{M}: T M \rightarrow M$. The vector fields $X^{C}$ and $X^{V}$ on $T M$ stand, respectively, for the complete lift and the vertical lift of a vector field $X$ on $M$. In natural coordinates $\left(x^{A}, \dot{x}^{A}\right)$ on $T M$ and for $X=X^{A} \partial / \partial x^{A}$ we get

$$
X^{C}=X^{A} \frac{\partial}{\partial x^{A}}+\dot{x}^{B} \frac{\partial X^{A}}{\partial x^{B}} \frac{\partial}{\partial \dot{x}^{A}} \quad \text { and } \quad X^{V}=X^{A} \frac{\partial}{\partial \dot{x}^{A}} .
$$

The Lie brackets of these vector fields are $\left[X^{C}, Y^{C}\right]=[X, Y]^{C},\left[X^{C}, Y^{V}\right]=[X, Y]^{V}$ and $\left[X^{V}, Y^{V}\right]=0$.

The notion of complete and vertical lifts also extends to functions and $(1,1)$ tensor fields, as follows. For a function $f$ on $M$, its lifts are the functions $f^{V}=f \circ \tau_{M}$ and $f^{C}=\dot{x}^{A}\left(\partial f / \partial x^{A}\right)$ on $T M$. Let $A$ be a $(1,1)$ tensor field on $M$. We may lift it to two $(1,1)$ tensor fields $A^{C}$ and $A^{V}$ on $T M$, as follows

$$
A^{C}\left(X^{V}\right)=(A(X))^{V}, \quad A^{C}\left(X^{C}\right)=(A(X))^{C}, \quad A^{V}\left(X^{V}\right)=0, \quad A^{V}\left(X^{C}\right)=(A(X))^{V} .
$$

More details on this can be found in [19], such as the following immediate properties:

$$
\mathcal{L}_{X^{V}} A^{C}=\left(\mathcal{L}_{X} A\right)^{V}, \quad \mathcal{L}_{X^{C}} A^{C}=\left(\mathcal{L}_{X} A\right)^{C}, \quad \mathcal{L}_{X^{V}} A^{V}=0, \quad \mathcal{L}_{X^{C}} A^{V}=\left(\mathcal{L}_{X} A\right)^{V} .
$$


Definition 1. A vector field $\Gamma$ on $T M$ is a second-order differential equations field (SODE in short) on $M$ if all its integral curves $\gamma: I \rightarrow T M$ are lifted curves, that is of the type $\gamma=\dot{c}$, for base integral curves $c: I \rightarrow M$.

As such, $\Gamma$ takes the form

$$
\Gamma=\dot{x}^{A} \frac{\partial}{\partial x^{A}}+f^{A}(x, \dot{x}) \frac{\partial}{\partial \dot{x}^{A}} .
$$

As a vector field on $T M$, a SODE is characterized by the property that $T \tau_{M} \circ \Gamma=\mathrm{id}_{M}$. A SODE $\Gamma$ on $M$ always exists, since a Riemannian metric on a (paracompact) manifold always exists, and we may consider its geodesic spray.

In what follows we will often express a SODE in terms of a frame of vector fields $Z_{A}=$ $Z_{A}^{B} \partial / \partial x^{B}$, which are not necessarily coordinate vector fields. In that context, we say that the quasi-velocities $\left(v^{A}\right)$ of a vector $v_{m} \in T_{m} M$ are the components of $v_{m}$ with respect to that basis, i.e., $v_{m}=v^{A} Z_{A}(m)$. Their relation to the standard fibre coordinates $\dot{x}^{A}$ is $\dot{x}^{A}=Z_{B}^{A}(m) v^{B}$.

In terms of the frame $\left\{Z_{A}\right\}$, the SODE $\Gamma$ takes the form

$$
\Gamma=v^{A} Z_{A}^{C}+F^{A} Z_{A}^{V},
$$

meaning that two SODEs $\Gamma_{1}$ and $\Gamma_{2}$ only differ in their coefficients $F_{1}^{A}$ and $F_{2}^{A}$. Let $c(t)=$ $\left(x^{A}(t)\right)$ be a base integral curve of $\Gamma$. The fibre coordinates of integral curves $\dot{c}(t)$ of $\Gamma$ in $T M$ may also be expressed in quasi-velocities as $\dot{c}(t)=v^{A}(t) Z_{A}(c(t))$. The functions $\left(x^{A}(t), v^{A}(t)\right)$ are then solutions of the equations

$$
\dot{x}^{A}(t)=Z_{B}^{A}(x(t)) v^{B}(t), \quad \dot{v}^{A}=F^{A}(x(t), v(t)) .
$$

Assume that $M$ comes equipped with a free and proper (left) action $\Phi: G \times M \rightarrow M$ of a Lie group $G$, such that $\pi: M \rightarrow \bar{M}=M / G$ is a principal fibre bundle. For each element $\xi$ in the Lie algebra $\mathfrak{g}$ we may obtain a fundamental vector field $\xi_{M}$ on $M$, defined by $\xi_{M}(m)=T \Phi_{m}(\xi)$ where, as usual $\Phi_{m}: G \rightarrow M$ denotes the map $\Phi(\cdot, m)$. In what follows it will be more convenient to write $\xi_{M}=\tilde{\xi}$ for a fundamental vector field on $M$. We will also assume that $G$ is connected. In that case, a vector field $X$ on $M$ is $G$-invariant if and only if $[X, \tilde{\xi}]=0$, for all $\xi \in \mathfrak{g}$.

There exist two ways to lift the action $\Phi$ to an action on $T M$. The first action is the $G$ action $\Phi^{T M}$ on $T M$, defined by $\Phi_{g}^{T M}=T\left(\Phi_{g}\right)$ where again we write $\Phi_{g}=\Phi(g, \cdot): M \rightarrow M$. With this action $\pi^{T M}: T M \rightarrow(T M) / G$, the so-called Atiyah bundle, is a principal $G$-bundle. Fundamental vector fields of this action are vector fields on $T M$ of the type $\tilde{\xi}^{C}$.

The tangent manifold $T G$ of a Lie group $G$ is also a Lie group. It may be identified with the semidirect product $G \times \mathfrak{g}$, and its Lie algebra with $\mathfrak{g} \times \mathfrak{g}$. The second action on $T M$ is the $T G$-action, given by $T \Phi: T G \times T M \rightarrow T M$. In the current trivialization, this action may be written as

$$
(g, \xi) \cdot v_{m}=T \Phi_{g}\left(v_{m}+\tilde{\xi}(m)\right) .
$$

With this action $(T M) /(T G)=T(M / G)$ and $T \pi: T M \rightarrow T(M / G)$ is also a principal bundle, but with structure group $T G$ (see, e.g., [11]). The fundamental vector fields that correspond to $T \Phi$ are linear combinations of the vector fields on $T M$ given by $(\xi, 0)_{T M}=\tilde{\xi}^{C}$ and $(0, \xi)_{T M}=\tilde{\xi}^{V}$.

To clarify the concepts we introduce later on, we will often use local coordinates $\left(x^{i}\right)$ on $\bar{M}=M / G$ and coordinates $\left(x^{A}\right)=\left(x^{i}, x^{a}\right)$ on $M$ that are adapted to the bundle $\pi$. On the tangent manifold, however, we will use quasi-velocities with respect to a specific frame. If $\left\{E_{a}\right\}$ is a basis for the (left) Lie algebra $\mathfrak{g}$, we will denote the fundamental vector fields (for the 
$G$-action on $M)$ by $\tilde{E}_{a}$. These vector fields span the vertical distribution of $\pi$, and their Lie brackets are given by $\left[\tilde{E}_{a}, \tilde{E}_{b}\right]=-C_{a b}^{c} \tilde{E}_{c}$, if we denote the structure constants of $\mathfrak{g}$ by $C_{a b}^{c}$.

We now assume that we have chosen a principal connection on $\pi$ (such a connection always exists). Let $X_{i}$ be the horizontal lifts, with respect to this connection, of the coordinate fields $\partial / \partial x^{i}$ on $M / G$. The fact that the horizontal lifts are $G$-invariant vector fields may be expressed as $\left[X_{i}, \tilde{E}_{a}\right]=0$. Given that

$$
\left[X_{i}^{C}, \tilde{E}_{a}^{\{C, V\}}\right]=\left[X_{i}, \tilde{E}_{a}\right]^{\{C, V\}}=0, \quad\left[X_{i}^{V}, \tilde{E}_{a}^{\{C, V\}}\right]=0
$$

we see that both $X_{i}^{C}$ and $X_{i}^{V}$ are invariant vector fields, for both the $G$-action on $T M$, and the $T G$-action on $T M$. The Lie bracket $\left[X_{i}, X_{j}\right]=R_{i j}^{a} \tilde{E}_{a}$ of these horizontal vector fields represents the curvature of the connection.

We will denote the quasi-velocities with respect to the frame $\left\{X_{i}, \tilde{E}_{a}\right\}$ as $\left(v^{i}, v^{a}\right)$. Actually, since the vector fields $X_{i}$ project, we may conclude that the quasi-velocities $v^{i}$ can be identified with the natural fibre coordinates on $M / G$, i.e., $v^{i}=\dot{x}^{i}$. Some further immediate properties are included in the list below (see, e.g., $[8,13]$ for more details):

$$
\begin{array}{llll}
X_{i}^{C}\left(v^{j}\right)=0, & X_{i}^{V}\left(v^{j}\right)=\delta_{i}^{j}, & X_{i}^{C}\left(v^{a}\right)=-R_{i j}^{a} v^{j}, & X_{i}^{V}\left(v^{a}\right)=0, \\
\tilde{E}_{a}^{C}\left(v^{i}\right)=0, & \tilde{E}_{a}^{V}\left(v^{i}\right)=0, & \tilde{E}_{a}^{C}\left(v^{b}\right)=C_{a c}^{b} v^{c}, & \tilde{E}_{a}^{V}\left(v^{b}\right)=\delta_{a}^{b} .
\end{array}
$$

A SODE $\Gamma$ takes the form

$$
\Gamma=v^{i} X_{i}^{C}+v^{a} \tilde{E}_{a}^{C}+F^{i} X_{i}^{V}+F^{a} \tilde{E}_{a}^{V}
$$

when expressed in this frame. It will be $G$-invariant when

$$
\left[\Gamma, \tilde{E}_{a}^{C}\right]=0 \quad \Leftrightarrow \quad \tilde{E}_{a}^{C}\left(F^{i}\right)=0, \quad \tilde{E}_{a}^{C}\left(F^{b}\right)=F^{c} C_{a c}^{b} .
$$

It is important to realize, however, that a SODE can never be $T G$-invariant since, besides the previous properties, it would also have to satisfy $\left[\Gamma, \tilde{E}_{a}^{V}\right]=0$, which is impossible because of the first term in

$$
\left[\Gamma, \tilde{E}_{a}^{V}\right]=-\tilde{E}_{a}^{C}-\tilde{E}_{a}^{V}\left(F^{i}\right) X_{i}^{V}-\left(\tilde{E}_{a}^{V}\left(F^{b}\right)+v^{c} C_{c a}^{b}\right) \tilde{E}_{b}^{V} .
$$

For this reason a SODE on $M$ can never be the horizontal lift of a principal connection on the $T G$-bundle $T \pi: T M \rightarrow T(M / G)$.

\section{Two lifted connections}

Assume that a principal connection on $\pi$ is given. There are many equivalent ways to represent this principal connection. For example, we may either consider it as being given by a $(1,1)$-tensor field $\omega$ on $M$ (its "vertical projection operator") or by a connection map $\varpi: T M \rightarrow \mathfrak{g}$. These two approaches are related as $\omega(X)(m)=\varpi \widetilde{(X(m)})(m)$. A third way to define a connection makes use of its horizontal lift. The horizontal lift of a vector field $\bar{X}$ on $\bar{M}$ is the unique vector field $\bar{X}^{h}$ on $M$ that projects on $\bar{X}$ and that is such that $\omega\left(\bar{X}^{h}\right)=0$. In what follows we will use these three definitions simultaneously.

The principal connection lifts to a principal connection on each of the principal bundles $\pi^{T M}$ and $T \pi$. In [13, Proposition 3], it is shown that the complete lift $\omega^{C}(\mathrm{a}(1,1)$ tensor field on $T M)$ represents a connection on the fibre bundle $T \pi: T M \rightarrow T(M / G)$. Moreover, since $\mathcal{L}_{\tilde{\xi}} \omega=0$, the properties of complete lifts lead to $\mathcal{L}_{\tilde{\xi}^{C}} \omega^{C}=0$ and $\mathcal{L}_{\tilde{\xi}^{V}} \omega^{C}=0$, from which we may conclude that this connection is principal with respect to the structure group $T G$. The idea of using the complete lift of a connection on a bundle $Y \rightarrow X$ to define a connection on $T Y \rightarrow T X$ comes from the paper [18] by Vilms, who used it in the context of vector bundles. For that reason, we will refer to $\omega^{C}$ as the Vilms connection. 
Definition 2. The Vilms connection of a principal connection $\omega$ on $\pi: M \rightarrow \bar{M}$ is the $T G$ principal connection on $T \pi: T M \rightarrow T \bar{M}$ whose vertical projection operator is given by the tensor field $\omega^{C}$.

The Vilms connection is characterized by its action on vector fields on $T M$, and therefore by

$$
\omega^{C}\left(X_{i}^{C}\right)=0, \quad \omega^{C}\left(X_{i}^{V}\right)=0, \quad \omega^{C}\left(\tilde{E}_{a}^{C}\right)=\tilde{E}_{a}^{C}, \quad \omega^{C}\left(\tilde{E}_{a}^{V}\right)=\tilde{E}_{a}^{V} .
$$

Proposition 1. Let $\omega$ be a principal connection on $\pi$. The horizontal lift $H$ of the Vilms connection can be characterized in terms of the horizontal lift $h$ of $\omega$, by means of the relations

$$
\left(\bar{X}^{C}\right)^{H}=\left(\bar{X}^{h}\right)^{C} \quad \text { and } \quad\left(\bar{X}^{V}\right)^{H}=\left(\bar{X}^{h}\right)^{V},
$$

for any vector field $\bar{X}$ on $\bar{M}$.

Proof. The horizontal lift of the Vilms connection maps a vector field $\bar{Y}$ on $T(M / G)$ onto a vector field $\bar{Y}^{H}$ on $T M$. Vector fields on $T(M / G)$ are functional combinations of the coordinate vector fields $\partial / \partial x^{i}$ and $\partial / \partial \dot{x}^{i}$. One may easily check that their horizontal lifts to vector fields on $T M$, for the Vilms connection, are

$$
\left(\frac{\partial}{\partial x^{i}}\right)^{H}=X_{i}^{C}, \quad\left(\frac{\partial}{\partial \dot{x}^{i}}\right)^{H}=X_{i}^{V}
$$

From this, one can deduce the statement in the proposition.

If we are given a SODE on $M / G, \bar{\Gamma}=\dot{x}^{i} \partial / \partial x^{i}+f^{i} \partial / \partial \dot{x}^{i}$, its horizontal lift is the vector field on $T M$ given by

$$
\bar{\Gamma}^{H}=\dot{x}^{i} X_{i}^{C}+f^{i} X_{i}^{V} .
$$

Since $\mathcal{L}_{\tilde{E}_{a}^{\{C, V\}}} \omega^{C}=0$, we have $\left[\tilde{E}_{a}^{\{C, V\}}, \bar{\Gamma}^{H}\right]=0$, which expresses that $\bar{\Gamma}^{H}$ is $T G$-invariant. Its $T G$-reduced vector field is, of course, $\bar{\Gamma}$. However, $\bar{\Gamma}^{H}$ is not a SODE on $M$ : Essentially we are missing the term " $v^{a} \tilde{E}_{a}^{C}$ ". To understand the part we are missing, we need to invoke a second connection.

The second connection is the "vertical lift" of $\omega$. By this we do not mean the $(1,1)$ tensor field $\omega^{V}$ we had defined above. We now need the interpretation of the connection on $\pi$ as the connection map $\varpi: T M \rightarrow \mathfrak{g}$.

Definition 3. The vertical connection of a principal connection $\varpi$ on $\pi: M \rightarrow \bar{M}$ is the $G$ principal connection on $\pi^{T M}: T M \rightarrow(T M) / G$ whose connection map $\varpi^{V}: T T M \rightarrow \mathfrak{g}$ is given by $\varpi^{V}=\tau_{M}^{*} \varpi$, with $\tau_{M}: T M \rightarrow M$.

For the corresponding interpretation of $\varpi^{V}$ as a $(1,1)$-form $\Omega$ on $T M$ one may calculate that

$$
\Omega\left(X_{i}^{C}\right)=0, \quad \Omega\left(X_{i}^{V}\right)=0, \quad \Omega\left(\tilde{E}_{a}^{C}\right)=\tilde{E}_{a}^{C}, \quad \Omega\left(\tilde{E}_{a}^{V}\right)=0 .
$$

It may easily be verified that this defines a principal connection on the $G$-bundle $\pi^{T M}$ : Among other properties, $\mathcal{L}_{\tilde{E}_{a}^{C}} \Omega=0$. The tensor field $\Omega$ clearly differs from the tensor field $\omega^{V}$, since, for example, $\Omega\left(\tilde{E}_{a}^{C}\right)=\tilde{E}_{a}^{C}$, but $\omega^{V}\left(\tilde{E}_{a}^{C}\right)=\tilde{E}_{a}^{V}$.

Assume that $\Gamma$ is a $G$-invariant SODE which reduces to the vector field $\hat{\Gamma}$ on $(T M) / G$. The vertical lift connection $\varpi^{V}$ can be used to reconstruct the integral curve $\gamma$ (through $v_{0}$ ) of $\Gamma$, starting from the integral curve $\hat{\gamma}$ (through $\pi^{T M}\left(v_{0}\right)$ ) of $\hat{\Gamma}$. This procedure is in fact valid for any principal bundle $N \rightarrow N / G$ with a given principal connection, not necessarily $N=T M$ (see, e.g., [13, Proposition 1], where it is explained in detail). Let $\hat{\gamma}^{\text {Hor }}$ be the horizontal lifted curve 
(by means of the principal connection $\varpi^{V}$ ) of $\hat{\gamma}$ through $v_{0}$. It is defined by the properties that it remains horizontal everywhere, that it goes through $v_{0}$ and that it projects on $\hat{\gamma}$. Let $\theta$ be the Maurer-Cartan form on $G$, i.e., $\theta\left(v_{g}\right)=T L_{g^{-1}} v_{g}$ for $v_{g} \in T_{g} G$. The reconstruction theorem states that if one solves the equation $\theta(\dot{g})=\varpi^{V}\left(\Gamma \circ \hat{\gamma}^{\text {Hor }}\right)$ (with $g(0)=e$ ) for a curve $g(t)$ in $G$, then the relation between the integral curves is given by $\gamma(t)=\Phi_{g(t)}^{T M} \hat{\gamma}^{\mathrm{Hor}}(t)$. If we write the SODE $\Gamma$ as before as $\Gamma=v^{i} X_{i}^{C}+v^{a} \tilde{E}_{a}^{C}+F^{i} X_{i}^{V}+F^{a} \tilde{E}_{a}^{V}$, then the reconstruction equation is of the form $(\theta(\dot{g}))^{a}=v^{a}$, where $v^{a}$ are the vertical quasi-velocities of $\hat{\gamma}^{\text {Hor }}$. It is clear that, in this respect, the reconstruction equation is essentially related to the term $v^{a} \tilde{E}_{a}^{C}$ of the SODE $\Gamma$. In what follows, we will often make use of this vector field, and we will denote it by $X_{\omega}$.

Proposition 2. Let $\Gamma_{0}$ be an arbitrary SODE on $M$. The vector field $X_{\omega}=\Omega\left(\Gamma_{0}\right)$ on $T M$ is

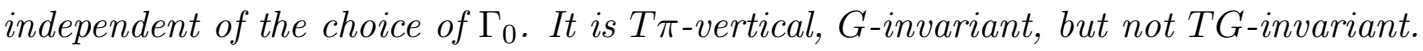

Proof. Since $\Gamma_{0}$ must take the form $v^{a} \tilde{E}_{a}^{C}+\cdots$, the vector field $\Omega\left(\Gamma_{0}\right)=v^{a} \tilde{E}_{a}^{C}$ is independent of the choice of the SODE $\Gamma_{0}$. It is clearly $T \pi$-vertical since $T \pi \circ \Omega=0$. It is also easy to see that this vector field is $G$-invariant, but not $T G$-invariant. Indeed, we have

$$
\left[X_{\omega}, \tilde{E}_{b}^{C}\right]=v^{a}\left[\tilde{E}_{a}^{C}, \tilde{E}_{b}^{C}\right]-\tilde{E}_{b}^{C}\left(v^{a}\right) \tilde{E}_{a}^{C}=0
$$

but, on the other hand,

$$
\left[X_{\omega}, \tilde{E}_{b}^{V}\right]=v^{a}\left[\tilde{E}_{a}^{C}, \tilde{E}_{b}^{V}\right]-\tilde{E}_{b}^{V}\left(v^{a}\right) \tilde{E}_{a}^{C}=-\left(v^{a} C_{a b}^{c} \tilde{E}_{c}^{V}+\tilde{E}_{b}^{C}\right)
$$

From its expression in quasi-velocities, it can be seen that a pointwise definition for the vector field $X_{\omega}$ is $X_{\omega}(v)=\widetilde{(\varpi(v))}(v)$, for all $v \in T M$.

\section{Un-reducing second-order systems}

We start with some general considerations for an arbitrary principal bundle $\mu: P \rightarrow P / G$.

Definition 4. A vector field $X \in \mathcal{X}(P)$ is an un-reduction of a vector field $\bar{X} \in \mathcal{X}(P / G)$ if all its integral curves project on those of $\bar{X}$.

This is actually equivalent with saying that $X$ and $\bar{X}$ are $\mu$-related, since for any integral curve $c(t)$ of $X$ the property in the definition means that $(\mu \circ c)(t)=(\bar{c} \circ \mu)(t)$, which, after differentiating, becomes

$$
T \mu \circ X=\bar{X} \circ \mu \text {. }
$$

For each principal connection $\varpi$ on $\mu$, the horizontal lift $\bar{X}^{h}$ is clearly an un-reduction. This shows that if we fix a principal connection, any un-reduction $X$ is of the type $X=\bar{X}^{h}+W$, where $W$ may be any $\mu$-vertical vector field on $P$.

We now turn to the case of interest, where $\mu=T \pi$ and $P=T M$ with structure group $T G$. For any vector field $\bar{X}$ on $T(M / G)$, the Vilms connnection $\omega^{C}$ on $T \pi$ generates an un-reduction $\bar{X}^{H}$. We will be interested in the case where either $\bar{X}$ or $X$ is a SODE.

Proposition 3. Let $\bar{\Gamma}$ be a vector field on $T(M / G)$, and let $\Gamma$ be an un-reduction of $\bar{\Gamma}$ on $T M$.

(1) If $\Gamma$ is a SODE on $M$, then $\bar{\Gamma}$ is a SODE on $M / G$.

(2) If $\bar{\Gamma}$ is a SODE on $M / G$, then $\Gamma$ is a SODE on $M$ if, and only if, $\varpi^{V} \circ \Gamma=\varpi$.

(3) If $\bar{\Gamma}$ is a SODE on $M / G$, then $\Gamma$ is a SODE on $M$ if, and only if, $\Omega(\Gamma)=X_{\omega}$. 
Proof. (1) The first statement follows from the commutative diagrams below. If $\Gamma$ is an unreduction and a SODE it satisfies $T T \pi \circ \Gamma=\bar{\Gamma}$ and $T \tau_{M} \circ \Gamma=\operatorname{Id}_{T M}$. After applying $T \pi$ on the last relation, we get $T\left(\pi \circ \tau_{M}\right) \circ \Gamma=\operatorname{Id}_{T(M / G)}$ and, from the diagram, the left hand side becomes $T \tau_{M_{G}} \circ T T \pi \circ \Gamma=T \tau_{M_{G}} \circ \bar{\Gamma}$, from which it follows that $\bar{\Gamma}$ is a SODE.
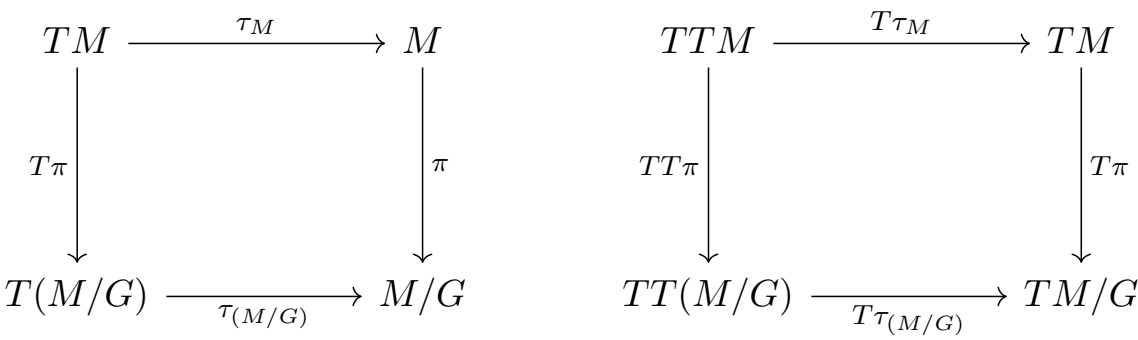

(2) The condition can be rewritten as $\varpi \circ T \tau_{M} \circ \Gamma=\varpi$. If $\Gamma$ is a SODE then $T \tau_{M} \circ \Gamma=$ $\operatorname{Id}_{T M}$ and the statement follows. Assume now that $\varpi \circ T \tau_{M} \circ \Gamma=\varpi$. We may use the principal connection to decompose each $w_{m} \in T_{m} M$ into its horizontal and vertical part as $\left(m, T \pi\left(w_{m}\right)\right)^{h}+\left(\varpi\left(w_{m}\right)\right)_{M}(m)$. For $w_{m}=T \tau_{M}\left(\Gamma\left(v_{m}\right)\right)$ we get, in view of the assumption that $\Gamma$ is an un-reduction of a $\operatorname{SODE} \bar{\Gamma}$ on $M / G$,

$$
\begin{aligned}
w_{m} & \left.=\left(m, T\left(\pi \circ \tau_{M}\right)\left(\Gamma\left(v_{m}\right)\right)\right)\right)^{h}+\left(\varpi\left(T \tau_{M}\left(\Gamma\left(v_{m}\right)\right)\right)\right)_{M}(m) \\
& \left.=\left(m, T\left(\tau_{M / G} \circ T \pi\right)\left(\Gamma\left(v_{m}\right)\right)\right)\right)^{h}+\left(\varpi\left(v_{m}\right)\right)_{M}(m) \\
& =\left(m, T\left(\tau_{M / G}\right)\left(\bar{\Gamma}\left(T \pi\left(v_{m}\right)\right)\right)\right)^{h}+\left(\varpi\left(v_{m}\right)\right)_{M}(m)=\left(m, T \pi\left(v_{m}\right)\right)^{h}+\left(\varpi\left(v_{m}\right)\right)_{M}(m) .
\end{aligned}
$$

From this it follows that $T \tau_{M}\left(\Gamma\left(v_{m}\right)\right)=w_{m}=v_{m}$, and that $\Gamma$ is a SODE on $M$.

(3) The relation between the connection map $\varpi$ of a connection, and its vertical projection $\omega$ is given by $\omega(X)(m)=\varpi \widetilde{(X(m))}(m)$. In the case of the vertical connection this becomes

$$
\left.\Omega(\Gamma)\left(v_{m}\right)=\left(\varpi^{V\left(\Gamma\left(v_{m}\right)\right.}\right)\right)^{C}\left(v_{m}\right) .
$$

If $\Gamma$ is an un-reduction of a SODE $\bar{\Gamma}$ then it will be SODE as well, in view of (2), if and only if $\Omega(\Gamma)\left(v_{m}\right)=\left(\widetilde{\varpi\left(v_{m}\right)}\right)^{C}\left(v_{m}\right)=X_{\omega}\left(v_{m}\right)$.

We may now introduce the following definition.

Definition 5. Let $\bar{\Gamma}$ be a SODE on $\bar{M}$ and $\omega$ be a principal connection on $\pi$. The primary un-reduced SODE of $(\bar{\Gamma}, \omega)$ is the SODE $\Gamma_{1}=\bar{\Gamma}^{H}+X_{\omega}$ on $M$.

In the frame $\left\{X_{i}, \tilde{E}_{a}\right\}, \Gamma_{1}$ takes the form $\Gamma_{1}=\bar{\Gamma}^{H}+\Gamma_{\omega}=v^{i} X_{i}^{C}+f^{i} X_{i}^{V}+v^{a} \tilde{E}_{a}^{C}+0 \tilde{E}_{a}^{V}$. The reason for calling $\Gamma_{1}$ an "un-reduced" SODE is given in the third observation below.

Proposition 4. Let $\bar{\Gamma}$ be a SODE on $\bar{M}$ and $\omega$ be a principal connection on $\pi$.

(1) The primary un-reduced SODE $\Gamma_{1}$ of $(\bar{\Gamma}, \omega)$ is a $G$-invariant vector field on $T M$, but is not TG-invariant.

(2) The SODE $\Gamma_{1}$ is the unique un-reduced SODE on $M$ that satisfies $\omega^{C}(\Gamma)=X_{\omega}$.

(3) The base integral curves c of $\Gamma_{1}$ project, via $\pi: M \rightarrow \bar{M}$, on base integral curves $\bar{c}$ of $\bar{\Gamma}$.

(4) $\Gamma_{1}$ is the unique SODE that projects on $\bar{\Gamma}$ and that has the property that it is tangent to the horizontal distribution of $\omega$. 
Proof. The first observation follows from the fact that $\bar{\Gamma}^{H}$ is $T G$-invariant, and therefore $\left[\Gamma_{1}, \tilde{\xi}^{\{C, V\}}\right]=\left[X_{\omega}, \tilde{\xi}^{\{C, V\}}\right]$. The second observation follows from the fact that the vector fields $X^{C}$ and $Y^{C}$ are $T \rho$-related if and only if $X$ and $Y$ are $\rho$-related. A similar observation holds for vertical lifts. One can easily prove this by considering the flows of the involved vector fields. In our case, we know that $\bar{\Gamma}^{H}$ and $\bar{\Gamma}$ are $T \pi$-related by definition, and that $\tilde{E}_{a}$ is $\pi$-related to zero, whence $X_{\omega}$ is $T \pi$-related to zero as well.

The observation that the SODE $\Gamma_{1}$ is $T \pi$-related to the SODE $\bar{\Gamma}$ means that its integral curves $\gamma$ in $T M$ project, via $T \pi$, on integral curves $\bar{\gamma}$ of $\bar{\Gamma}$ in $T \bar{M}$. Since both vector fields are SODEs, their integral curves are lifted curves. We conclude from this that the base integral curves $c$ of $\Gamma_{1}$ project, via $\pi: M \rightarrow \bar{M}$, on base integral curves $\bar{c}$ of $\bar{\Gamma}$.

Horizontal vectors, expressed in quasi-velocities as $v_{m}=v^{i} X_{i}(m)+v^{a} \tilde{E}_{a}^{C}(m)$, have the property that $v^{a}=0$. Let's write $\Gamma_{1}$ for now as $\Gamma_{1}=v^{i} X_{i}^{C}+f^{i} X_{i}^{V}+v^{a} \tilde{E}_{a}^{C}+F^{a} \tilde{E}_{a}^{V}$. For the last property we need to show that $\Gamma_{1}\left(v^{b}\right)=0$ is equivalent with $F^{a}=0$. This follows easily from the properties $X_{i}^{C}\left(v^{b}\right)=-R_{i j}^{b} v^{j}, X_{i}^{V}\left(v^{a}\right)=0, \tilde{E}_{a}^{C}\left(v^{b}\right)=C_{a c}^{b} v^{c}$ and $\tilde{E}_{a}^{V}\left(v^{b}\right)=\delta_{a}^{b}$.

Integral curves of $\bar{\Gamma}$ satisfy $\ddot{x}^{i}=f^{i}(x, \dot{x})$. For $\Gamma_{1}=\bar{\Gamma}^{H}+X_{\omega}=v^{i} X_{i}^{C}+f^{i} X_{i}^{V}+v^{a} \tilde{E}_{a}^{C}+0 \tilde{E}_{a}^{V}$, the integral curves satisfy, among other, $\ddot{x}^{i}=f^{i}(x, \dot{x})$ and $\dot{v}^{a}=0$. The remaining equation is the "reconstruction equation" $(\theta(\dot{g}))^{a}=v^{a}$, where $\theta$ is the Maurer-Cartan form (see the paragraph above Proposition 2).

From the first statement in Proposition 4, the one which says that $\Gamma_{1}$ is $G$-invariant, we may conclude that $\Gamma_{1}$ can be reduced to a vector field on $(T M) / G$. This vector field is, obviously, not the same as the SODE $\bar{\Gamma}$, which is a vector field on $T(M / G)$. Vector fields on $(T M) / G$ play, however, an essential role in so-called Lagrange-Poincaré reduction. The Euler-Lagrange equations of a $G$-invariant regular Lagrangian produce such a $G$-invariant SODE on $M$, and the integral curves of its reduced vector field on $(T M) / G$ satisfy what are called the LagrangePoincaré equations. In this sense, one may think of the un-reduction of [5], which heavily relies on Lagrange-Poincaré reduction, as a process that compares the reduced vector field on $(T M) / G$ with the vector field $\bar{\Gamma}$ on $T(M / G)$.

Let $\bar{c}$ be a curve in $M / G$ which goes at $t=0$ through a point $\bar{m}$. Consider a point $m \in M$ with $\pi(m)=\bar{m}$. The horizontal lift of $\bar{c}$ at $m$ is the unique curve $\bar{c}_{m}^{h}$ which is defined by the following three properties: (1) it projects on $\bar{c}$ for all $t,(2)$ it satisfies $\bar{c}_{m}^{h}(0)=m,(3)$ it has only horizontal tangent vectors $\dot{\bar{c}}_{m}^{h}(t)$.

Proposition 5. The base integral curves of the primary un-reduced SODE $\Gamma_{1}$ of $(\bar{\Gamma}, \omega)$ through horizontal initial vectors are horizontal lifts of base integral curves of $\bar{\Gamma}$.

Proof. Consider an initial value $\bar{m}$ in $M / G$ and an initial tangent vector $\bar{v}=v_{0}^{i} \partial /\left.\partial x^{i}\right|_{\bar{m}}$, and their corresponding base integral curve $\bar{c}_{\bar{m}, \bar{v}}(t)$ of $\bar{\Gamma}$. Let $m \in M$ be such that $\pi(m)=\bar{m}$, and consider the vector $v:=h(m, \bar{v})$, the $\omega$-horizontal lift of $\bar{v}$ to $m$. In the frame $\left\{X_{i}, \tilde{E}_{a}\right\}$, we may write $v=v_{0}^{i} X_{i}(m)+0 \tilde{E}_{a}(m)$. With the initial values $(m, v)$, the base integral curve $c_{m, v}(t)$ of $\Gamma$ satisfies $\pi\left(c_{m, v}(t)\right)=\bar{c}_{\bar{m}, \bar{v}}(t)$ and also $v^{a}(t)=0$ (with this particular initial value). The solution of the remaining equation is then $\theta(\dot{g})=0$, from which we see that $g(t)$ remains constant.

From the fact that $v^{a}(t)=0$, we conclude that the velocity $\dot{\bar{c}}_{m}^{h}(t)$ remains horizontal throughout. Given that also (1) and (2) are satisfied, we may conclude that $c_{m, v}=\left(\bar{c}_{\bar{m}, \bar{v}}\right)_{m}^{h}$.

If we had taken a different initial condition, say $v^{a}(0)=\xi^{a}$, we would get $v^{a}(t)=\xi^{a}$, and the corresponding curve $c$ would not be a horizontal lift.

From this proposition, we may derive an "un-reduction algorithm". Consider a SODE $\bar{\Gamma}$ on $\bar{M}$, a principal connection on $M \rightarrow \bar{M}$ and their corresponding "lifted" primary un-reduced SODE $\Gamma_{1}=X_{\omega}+(\bar{\Gamma})^{H}$. Calculate its integral curves $c(t)$ in $M$, but only for the very specific initial values we gave above: the initial velocity has to be horizontal. We have just shown that 
these curves are in fact the horizontal lifts of the curves $\bar{c}$ that we really want to know. If we project them down, we get the desired curves $\bar{c}$. Note that any field of initial velocities works as well: we could compute the integral curves of $\Gamma_{1}$ for any set of initial conditions, and then project them in view of Proposition 4.

Other than the primary un-reduced SODE, there are many more SODEs that have the property that (some of) their integral curves project onto those of $\bar{\Gamma}$. All other un-reduction SODEs are of the form $\Gamma_{2}=\Gamma_{1}+V$, where $V$ is $T \tau$-vertical and $T \pi$-vertical. Since $\Gamma_{1}, X_{i}^{V}$ and $\tilde{E}_{a}^{V}$ are $T \pi$-related to $\bar{\Gamma}, \partial / \partial \dot{x}^{i}$ and 0 , respectively, this means that $V$ may be any vector field on $T M$ of the type $V=V^{a} \tilde{E}_{a}^{V}$, which means that it is a vector field with $\Omega(V)=0$.

Proposition 6. Let $\bar{\Gamma}$ be a SODE on $M / G$. Any vector field $\Gamma_{2}=\Gamma_{1}+V$, where $V$ is $T \pi$ vertical and such that $\Omega(V)=0$, is a SODE which has the property that its base integral curves project on those of $\bar{\Gamma}$.

The full expression of $\Gamma_{2}$ is $\Gamma_{2}=v^{i} X_{i}^{C}+f^{i} X_{i}^{V}+v^{a} \tilde{E}_{a}^{C}+V^{a} \tilde{E}_{a}^{V}$. Its integral curves satisfy $\ddot{x}^{i}=f^{i}$ and $\dot{v}^{a}=V^{a}$.

If we only consider vector fields $\Gamma_{2}$ that are $G$-invariant, then, since we know that $\Gamma_{1}$ is $G$-vertical, the coefficients $V^{a}$ need to satisfy $\tilde{E}_{a}^{C}\left(V^{b}\right)=C_{a d}^{b} V^{d}$.

With the above, we have characterized all $G$-invariant SODEs $\Gamma_{2}$ which have the property that all their integral curves project to those of $\bar{\Gamma}$. The systems that appear in [5] therefore belong to the SODEs we have discussed in this section, see Section 6 for more details. In Section 8, we mention a different class of SODEs for which only a subclass of its integral curves project.

\section{A non-Lagrangian example}

We will now consider an example where the dynamics is not of Lagrangian nature, and therefore falls out of the scope of [5]. This section intends to clarify some of the concepts we have introduced in the previous ones. First, we recall some well known facts. In general, if $\left\{Z_{A}\right\}$ is a frame on a manifold $M$, with quasi-velocities $v^{A}$, and if $\nabla$ is a linear affine connection with coefficients $\nabla_{Z_{A}} Z_{B}=\gamma_{A B}^{D} Z_{D}$, then its quadratic spray is the SODE given by $v^{A} Z_{A}^{C}-$ $\gamma_{A B}^{D} v^{A} v^{B} Z_{D}^{V}$. The base integral curves of this spray satisfy: $\dot{v}^{D}=-\gamma_{A B}^{D} v^{A} v^{B}$. This construction applies in particular to the case where the connection is the Levi-Civita connection of some (pseudo-)Riemannian metric on $M$.

Consider a connected Lie group $G$, with its Lie algebra $\mathfrak{g}$ given by the Lie algebra of leftinvariant vector fields. The canonical affine connection on a Lie group $G$ can be defined by its action on left-invariant vector fields:

$$
\nabla_{\zeta_{G}^{L}} \eta_{G}^{L}=\frac{1}{2}[\zeta, \eta]_{G}^{L}
$$

where $\zeta_{G}^{L}, \eta_{G}^{L}$ stand for the left-invariant vector fields of $\zeta, \eta \in \mathfrak{g}$.

Among other properties, $\nabla$ has vanishing torsion; see [14] for a detailed discussion of this and other aspects of $\nabla$. We denote its quadratic spray by $\Gamma_{G}$. If the Lie-algebra $\mathfrak{g}$ is (semi-simple or) compact then its Killing form defines a (pseudo-)Riemannian metric on $G$ for which the canonical connection is its Levi-Civita connection. In other cases, although the canonical spray is a quadratic spray, the canonical connection may not be metrical and, moreover, its spray need not even be variational (see, e.g., [17] for some occurrences of this situation). It is also well-known that if the canonical connection is the Levi-Civita of some left-invariant Riemannian metric, then that metric is necessarily also right-invariant (see [15]). The base integral curve of $\Gamma_{G}$ through $g$ and $\zeta \in \mathfrak{g}$ (when $T G$ is left trivialized as $G \times \mathfrak{g}$ ) is given by $t \mapsto g \exp (t \zeta)$. This is easy to see, as follows. 
The connection coefficients with respect to the frame $\left\{\left(E_{A}\right)_{G}^{L}\right\}$ of left-invariant vector fields are $\gamma_{A B}^{D}=\frac{1}{2} C_{A B}^{D}$, which are skew-symmetric. The spray is then $\Gamma_{G}=v_{L}^{A}\left(\left(E_{A}\right)_{G}^{L}\right)^{C}$ (with no $\left(\left(E_{A}\right)_{G}^{L}\right)^{V}$ component) and the equation for its base integral curves is $\dot{v}_{L}^{A}=0$, and therefore $v_{L}^{A}(t)=\zeta^{A}$, for some constants $\zeta^{A}$. Since $v_{L}^{A}$ are the quasi-velocities in the frame of left-invariant vector fields, they are precisely the components of $\dot{g}$ left-translated to the Lie algebra, i.e., we have $g^{-1} \dot{g}=\zeta$. The solution of $\left(g^{-1} \dot{g}\right)^{A}=\zeta^{A}$ with $g(0)=e$ is precisely the one-parameter group of $\zeta$. The rest of the argument relies on the symmetry of $\Gamma_{G}$.

Note that, from the expression $\Gamma_{G}=v_{L}^{A}\left(E_{A}\right)_{L}^{C}$, we may also write that $\Gamma_{G}\left(v_{g}\right)=\left(\zeta_{G}^{L}\right)^{C}\left(v_{g}\right)$, where $\zeta=T L_{g^{-1}} v_{g}=v^{A} E_{A}$.

Consider now a (closed) normal subgroup $N$ of $G$, and consider its right action on $G$. Then $K=G / N$ is again a Lie group, where multiplication is given by $\left(g_{1} N\right)\left(g_{2} N\right)=\left(g_{1} g_{2}\right) N$, where $g N$ denotes the left coset. We will write $\pi: G \rightarrow K=G / N$ for the projection and $\mathfrak{g}, \mathfrak{n}$ and $\mathfrak{k}$ for the corresponding Lie algebras.

Since $K$ is a Lie group, it comes with its own canonical connection $\nabla^{K}$. We wish to show that the sprays $\Gamma_{K}$ and $\Gamma_{G}$ of the two connections are related by means of an un-reduction process. Recall that, if that is the case, we only need to introduce coordinates on $G$ (and $N$ ) to be able to write down integral curves on $K$.

To start the un-reduction process we need a principal $N$-connection on $\pi: G \rightarrow K$. Such a connection is, for example, available if we consider on $\mathfrak{g}$ an $\operatorname{Ad}_{N}$-invariant inner product. (Remark that we do not assume that the inner product is $\mathrm{Ad}_{G}$-invariant. But, if that were the case, then it would generate a bi-invariant metric on $G$, whose Levi-Civita connection was $\nabla^{G}$.) The orthogonal complement $\mathfrak{m}$ of $\mathfrak{n}$ with respect to this inner product is then $\operatorname{Ad}_{N}$-invariant. In effect, this means that $N$ is reductive. We will write $\mathfrak{g}=\mathfrak{m} \oplus \mathfrak{n}$. In these notations $T_{e} \pi(\mathfrak{g})=\mathfrak{k}$. Consider the short exact sequence

$$
0 \rightarrow \mathfrak{n} \rightarrow \mathfrak{g} \rightarrow \mathfrak{k} \rightarrow 0 .
$$

Its splitting $\mathfrak{k} \rightarrow \mathfrak{g}$ with image $\mathfrak{m}$ will be denoted by $s$. From the property that $N$ is normal we get that $[\mathfrak{n}, \mathfrak{n}] \subset \mathfrak{n}$ and $[\mathfrak{n}, \mathfrak{m}] \subset \mathfrak{n}$ (i.e., $\mathfrak{n}$ is an ideal of $\mathfrak{g}$ ). From the fact that $\mathfrak{m}$ is $\operatorname{Ad}_{N}$-invariant, we also get that $[\mathfrak{n}, \mathfrak{m}] \subset \mathfrak{m}$, and therefore $[\mathfrak{n}, \mathfrak{m}]=\{0\}$. Remark that we do not know much about $[\mathfrak{m}, \mathfrak{m}]$ (its vertical part is related to the curvature of the connection we will introduce next).

Let $P_{\mathfrak{m}}: \mathfrak{g} \rightarrow \mathfrak{m}$ and $P_{\mathfrak{n}}: \mathfrak{g} \rightarrow \mathfrak{n}$ denote the projections on $\mathfrak{m}$ and $\mathfrak{n}$, respectively. We may associate an $N$-principal connection on $\pi: G \rightarrow K$ to the decomposition $\mathfrak{g}=\mathfrak{m} \oplus \mathfrak{n}$ by means of the connection map $\varpi\left(v_{g}\right)=P_{\mathfrak{n}}\left(T L_{g^{-1}} v_{g}\right)$. To see that this is a connection, recall that the infinitesimal generators of the $N$-action are given by the left-invariant vector fields $\eta_{G}^{L}$ on $G$, associated to $\eta \in \mathfrak{n}$. Given that $\operatorname{Ad}_{n} \circ P_{\mathfrak{n}}=P_{\mathfrak{n}} \circ \operatorname{Ad}_{n}$ (where $n \in N$ ) we may easily verify that indeed $\varpi\left(\eta_{G}^{L}(g)\right)=\eta$, for $\eta \in \mathfrak{n}$, and $\varpi\left(T R_{n} v_{g}\right)=\operatorname{Ad}_{n^{-1}}\left(\varpi\left(v_{g}\right)\right)$.

Consider now, for $\xi \in \mathfrak{k}$, the vector field $\xi_{K}^{L}$ on $K$. We will show that its horizontal lift, with respect to the connection $\varpi$, is the left-invariant vector field $(s \xi)_{G}^{L}$. It is easy to see that this vector field is $N$-invariant, since for all infinitesimal generators of the action, $\left[\eta_{G}^{L},(s \xi)_{G}^{L}\right]=$ $[\eta, s \xi]_{G}^{L}=0$ for all $\eta \in \mathfrak{n}$. Moreover, $(s \xi)_{G}^{L}$ projects on $\xi_{L}^{K}$, since

$$
T \pi\left((s \xi)_{G}^{L}(g)\right)=T \pi\left(T L_{g}(s \xi)\right)=T L_{g N}((\pi \circ s)(\xi))=T L_{g N}(\xi)=\xi_{L}^{K}(\pi(g)) .
$$

Finally, $(s \xi)_{G}^{L}$ is horizontal, since $\varpi\left((s \xi)_{G}^{L}(g)\right)=P_{\mathfrak{n}}\left(T L_{g^{-1}}\left(T L_{g}(s \xi)\right)\right)=P_{\mathfrak{n}}(s \xi)=0$.

Proposition 7. Let $N$ be a normal subgroup of a Lie group $G$ and consider the principal connection $\varpi$ associated to an $\operatorname{Ad}_{N}$-invariant inner product on $\mathfrak{g}$. Then, the canonical spray $\Gamma_{G}$ on $G$ is the primary un-reduced $S O D E$ of the canonical spray $\Gamma_{K}$ on $K=G / N$ and the connection $\varpi$. 
Proof. We first derive an expression for the Vilms-horizontal lift of the spray $\Gamma_{K}$. Consider a given $v_{g} \in T_{g} G$, and let $v_{k}=T \pi\left(v_{g}\right) \in T_{\pi(g)} K$. We will also use the notations $\zeta=T L_{g^{-1}} v_{g}$ and $\xi=T L_{k^{-1}} v_{k}$. By construction, $\Gamma_{K}\left(v_{k}\right)=\left(\xi_{L}^{K}\right)^{C}\left(v_{k}\right)$. Since the complete lifts on $G$ and $K$ commute with the two connections (on the one hand the Vilms-horizontal lift, and on the other hand the horizontal lift, see Proposition 1) we easily get that

$$
\begin{aligned}
\left(\Gamma_{K}\right)^{H}\left(v_{g}\right) & =\left(\left((\xi)_{K}^{L}\right)^{h}\right)^{C}\left(v_{g}\right)=\left((s \xi)_{G}^{L}\right)^{C}\left(v_{g}\right)=\left(\left(s\left(T L_{k^{-1}} T \pi\left(v_{g}\right)\right)\right)_{G}^{L}\right)^{C}\left(v_{g}\right) \\
& =\left(\left(s\left(T\left(\pi \circ L_{g^{-1}}\right)\left(v_{g}\right)\right)\right)_{G}^{L}\right)^{C}\left(v_{g}\right)=\left(\left((s \circ T \pi)\left(L_{g^{-1}}\left(v_{g}\right)\right)\right)_{G}^{L}\right)^{C}\left(v_{g}\right) \\
& =\left(\left(P_{\mathfrak{m}} \zeta\right)_{G}^{L}\right)^{C}\left(v_{g}\right) .
\end{aligned}
$$

On the other hand, we have already stated that, in the current notations,

$$
X_{\omega}\left(v_{g}\right)=\left(\left(\varpi\left(v_{g}\right)\right)_{G}^{L}\right)^{C}\left(v_{g}\right)=\left(\left(P_{\mathfrak{n}} \zeta\right)_{G}^{L}\right)^{C}\left(v_{g}\right) .
$$

Together, we get

$$
\left(\Gamma_{K}\right)^{H}\left(v_{g}\right)+X_{\omega}\left(v_{g}\right)=\left(\left(P_{\mathfrak{m}} \zeta\right)_{G}^{L}\right)^{C}\left(v_{g}\right)+\left(\left(P_{\mathfrak{n}} \zeta\right)_{G}^{L}\right)^{C}\left(v_{g}\right)=\left((\zeta)_{G}^{L}\right)^{C}\left(v_{g}\right),
$$

which is exactly $\Gamma_{G}\left(v_{g}\right)$.

Example: The general linear group as a bundle over $\mathbb{R}$. In the set $M_{n \times n}(\mathbb{R})$ of $n \times n$ real matrices we consider the Lie groups

$$
\begin{aligned}
& \mathrm{GL}^{+}(n)=\left\{A \in M_{n \times n}(\mathbb{R}): \operatorname{det}(A)>0\right\}, \\
& \mathrm{SL}(n)=\left\{A \in M_{n \times n}(\mathbb{R}): \operatorname{det}(A)=1\right\} .
\end{aligned}
$$

The group $\operatorname{SL}(n)$ is a normal subgroup of $\operatorname{GL}(n)^{+}$. This follows from the fact that it is the kernel of the Lie group homomorphism det: $\mathrm{GL}^{+} \rightarrow \mathbb{R}^{+}$, where $\mathbb{R}^{+}$is the multiplicative group of positive real numbers. In particular, the determinant induces an isomorphism:

$$
\varphi: \mathrm{GL}^{+}(n) / \mathrm{SL}(n) \rightarrow \mathbb{R}^{+} \text {. }
$$

The situation is summarized in the following diagram:

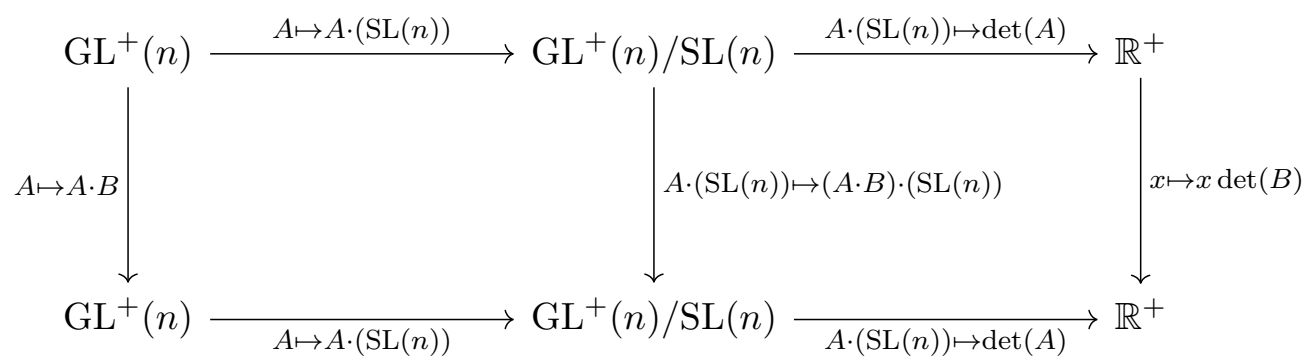

We can therefore think of $\mathrm{GL}^{+}(n)$ as a principal $\mathrm{SL}(n)$-bundle over $\mathbb{R}^{+}$, where $\mathrm{SL}(n)$ acts on the right on $\mathrm{GL}^{+}(n)$ and with the projection given by $\pi(A)=\operatorname{det}(A)$. We also observe that the tangent of the projection $\pi$ at Id (the $n \times n$ identity matrix) is $T_{\mathrm{Id}} \pi=$ trace.

The Lie algebra of $\mathrm{GL}^{+}(n)$ is $\mathfrak{g l}(n)$ and the Lie algebra $\mathfrak{s l}(n)$ of $\mathrm{SL}(n)$ consists of the real $n \times n$ traceless matrices. We consider the inner product on $\mathfrak{g l}(n)$ given by $\langle A, B\rangle_{\mathfrak{g l}(n)}=\operatorname{trace}\left(A B^{-1}\right)$. Since the trace is invariant under conjugation, $\langle\cdot, \cdot\rangle_{\mathfrak{g l}(n)}$ defines and $\operatorname{Ad}_{\mathrm{SL}(n)}$-invariant inner product on $\mathfrak{g l}(n)$. Note that the identity matrix Id is orthogonal to $\mathfrak{s l}(n)$, and therefore $\langle\cdot, \cdot\rangle_{\mathfrak{g l}(n)}$ 
induces the splitting $\mathfrak{g l}(n)=\mathfrak{s l}(n) \oplus\langle\mathrm{Id}\rangle$. The associated map $s: \mathbb{R} \rightarrow \mathfrak{g l}(n)$ is given by $s(\lambda)=$ $\left(\frac{\lambda}{n}\right)$ Id. The situation is as follows:

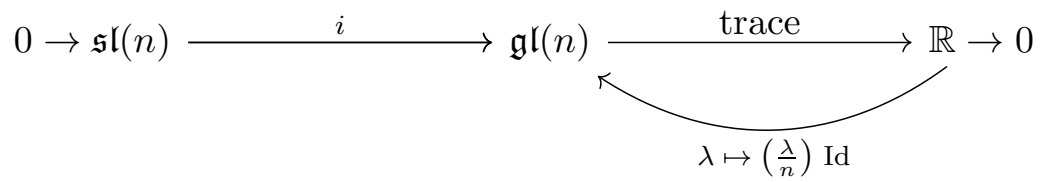

The horizontal space of the connection $\varpi$ is therefore given by left translation of the identity. A vector $v_{A}$ in $T_{A}\left(\mathrm{GL}^{+}(n)\right)$ is horizontal if it is of form $v_{A}=\mu A$, where $\mu \in \mathbb{R}$ (we identify the tangent space to a vector space with the vector space itself). The horizontal lift of the tangent vector $\lambda \in T_{x} \mathbb{R}^{+}$to $A \in \pi^{-1}(x)$ is given by the vector $v_{A}=\left(\frac{\lambda}{x n}\right) A$. Indeed, this vector is horizontal and it projects back, since $\pi \circ L_{A^{-1}}=L_{x^{-1}} \circ \pi$, and thus

$$
T \pi\left(v_{A}\right)=T L_{x} \circ T \pi\left(A^{-1} v_{A}\right)=x \operatorname{trace}\left(A^{-1} v_{A}\right)=\lambda .
$$

We will use $x$ for the coordinate on $\mathbb{R}^{+}$. When written in terms of vector fields, we may see from the above that, e.g., the horizontal lift of the coordinate vector field $\partial / \partial x$ on $\mathbb{R}^{+}$is the vector field defined by the map $X: \mathrm{GL}^{+}(n) \rightarrow \mathrm{GL}^{+}(n): A \mapsto \frac{1}{n \operatorname{det} A} A$. We may also write $X=\frac{1}{n x} \mathrm{id}$, where id stands for the vector field given by the identity map on $\mathrm{GL}^{+}(n)$.

We will write $\bar{\Gamma}$ and $\Gamma$ for the canonical sprays on $\mathbb{R}^{+}$and $\mathrm{GL}^{+}(n)$, respectively. The element $1 \in \mathbb{R}$ is a basis for the Lie algebra of $\mathbb{R}^{+}$. Its left-invariant vector field on $\mathbb{R}^{+}$is then $x \partial / \partial x$. If we use natural coordinates $(x, \dot{x}) \in T \mathbb{R}^{+}$, the quasi-velocity with respect to this vector field is then $w=\dot{x} / x$. Therefore, the canonical spray $\bar{\Gamma}$ on $\mathbb{R}^{+}$is

$$
\bar{\Gamma}=w\left(x \frac{\partial}{\partial x}\right)^{C}=\dot{x} \frac{\partial}{\partial x}+\frac{\dot{x}^{2}}{x} \frac{\partial}{\partial \dot{x}} .
$$

Due to the properties in Proposition 1, its Vilms horizontal lift is

$$
\bar{\Gamma}^{H}=\dot{x}\left(\left(\frac{\partial}{\partial x}\right)^{h}\right)^{C}+\frac{\dot{x}^{2}}{x}\left(\left(\frac{\partial}{\partial x}\right)^{h}\right)^{V}=\dot{x} X^{C}+\frac{\dot{x}^{2}}{x} X^{V} .
$$

But, $X=\frac{1}{n x}$ id. Given that in general $(f Y)^{C}=f Y^{C}+\dot{f} Y^{V}$, we may also write

$$
\bar{\Gamma}^{H}=\frac{\dot{x}}{n x}(\mathrm{id})^{C}-\frac{\dot{x}^{2}}{n x^{2}}(\mathrm{id})^{V}+\frac{\dot{x}^{2}}{n x^{2}}(\mathrm{id})^{V}=\frac{\dot{x}}{n x}(\mathrm{id})^{C} .
$$

Consider now a basis $\left\{E_{a}\right\} \cup \operatorname{Id}$ of $\mathfrak{g l}(n)\left(a=1, \ldots, n^{2}-1\right)$. It is clear that the fundamental vector field $(\mathrm{Id})_{\mathrm{GL}^{+}(n)}$ is simply the vector field id on $\mathrm{GL}^{+}(n)$. If $\left\{v^{a}, v\right\}$ denote the quasivelocities w.r.t. the frame $\left\{\tilde{E}_{a}\right.$, id $\}$ then we have that $v=\frac{\dot{x}}{n x}$. In the spray $\Gamma$,

$$
\Gamma=v^{a} \tilde{E}_{a}^{C}+v(\mathrm{id})^{C},
$$

we recognize therefore in the first term the vector field $X_{\omega}$, and in the last term the Vilms horizontal lift $\bar{\Gamma}^{H}$.

\section{Un-reducing Lagrangian systems}

We now specialize to the case where the SODE $\bar{\Gamma}$ is the Lagrangian vector field of a regular Lagrangian $\ell$ (a smooth function on $T \bar{M}$ ). The vector field $\bar{\Gamma}$ on $T \bar{M}$ is then completely determined by two facts: (1) it is a SODE, (2) it satisfies

$$
\bar{\Gamma}\left(\frac{\partial \ell}{\partial \dot{x}^{i}}\right)-\frac{\partial \ell}{\partial x^{i}}=0 .
$$


Written in coordinates, this characterizations says that if we write, in general, $\bar{\Gamma}=X^{i} \partial / \partial x^{i}+$ $f^{i} \partial / \partial \dot{x}^{i}$ for a vector field on $T \bar{M}$, then, from (1) we know that $X^{i}=\dot{x}^{i}$, and from (2) we get that $f^{i}$ is determined by the Euler-Lagrange equations, when written in normal form. We will translate property (2) into one that the primary un-reduced SODE $\Gamma_{1}=X_{\omega}+\bar{\Gamma}^{H}$ satisfies.

First, we recall the following observations for vector fields on a principal $K$-bundle $p: Q \rightarrow$ $Q / K$. When a vector field $W$ on $Q$ is $K$-invariant, the relation $\bar{W} \circ p=T p \circ W$ uniquely defines its reduced vector field $\bar{W}$ on $Q / K$. Likewise, if $F: Q \rightarrow \mathbb{R}$ is a $K$-invariant function on $Q$ it can be reduced to a function $f: Q / K \rightarrow \mathbb{R}$ with $f \circ p=F$. The relation between these objects can easily be seen to be

$$
W(F)=W(f \circ p)=\bar{W}(f) \circ p,
$$

which says that the function $\bar{W}(f)$ is the reduced function on $Q / K$ of the invariant function $W(F)$ on $Q$. We will use this for the case where $Q=T M$ and $K=T G$, so that $Q / K=T \bar{M}$ and $p=T \pi$.

Given $\ell$, we may define a function $L^{H}$ on $T M$ by $L^{H}(v):=\ell(T \pi(v))$. This function is not a regular Lagrangian on $M$. But it is a $T G$-invariant function, which means that $\tilde{E}_{a}^{C}\left(L^{H}\right)=$ $0=\tilde{E}_{a}^{V}\left(L^{H}\right)$. Its $T G$-reduced function is $\ell$. The vector fields $X_{i}^{C}, X_{i}^{V}, \bar{\Gamma}^{H}$ are all $T G$-invariant (since $\left[\tilde{E}_{a}^{\{C, V\}}, X_{i}^{C}\right]=0$ and similar for $X_{i}^{V}$ and $\bar{\Gamma}^{H}$ ), and their $T G$-reduced vector fields on $\bar{M}$ are $\partial / \partial x^{i}, \partial / \partial \dot{x}^{i}$ and $\bar{\Gamma}$, respectively. From the relation above, we get that

$$
X_{i}^{C}\left(L^{H}\right)=\frac{\partial \ell}{\partial x^{i}} \circ T \pi, \quad X_{i}^{V}\left(L^{H}\right)=\frac{\partial \ell}{\partial \dot{x}^{i}} \circ T \pi,
$$

and therefore, if $\bar{\Gamma}$ satisfies the Euler-Lagrange equation, we obtain that $\bar{\Gamma}^{H}$ satisfies:

$$
\bar{\Gamma}^{H}\left(X_{i}^{V}\left(L^{H}\right)\right)-X_{i}^{C}\left(L^{H}\right)=0 .
$$

We also know that $X_{i}^{V}\left(L^{H}\right)$ is a $G$-invariant function (this follows from $\left[\tilde{E}_{a}^{C}, X_{i}^{V}\right]\left(L^{H}\right)=0$ ), and therefore $X_{\omega}\left(X_{i}^{V}\left(L^{H}\right)\right)=0$. So, we may also write

$$
\Gamma_{1}\left(X_{i}^{V}\left(L^{H}\right)\right)-X_{i}^{C}\left(L^{H}\right)=0,
$$

or, if $\bar{X}^{h}=\bar{X}^{i} X_{i}$ is an arbitrary $\omega$-horizontal lift of a vector field $\bar{X}=\bar{X}^{i} \partial / \partial x^{i}$ on $\bar{M}$,

$$
\Gamma_{1}\left(\left(\bar{X}^{h}\right)^{V}\left(L^{H}\right)\right)-\left(\bar{X}^{h}\right)^{C}\left(L^{H}\right)=0,
$$

or, equivalently,

$$
\Gamma_{1}\left(\left(\bar{X}^{V}\right)^{H}\left(L^{H}\right)\right)-\left(\bar{X}^{C}\right)^{H}\left(L^{H}\right)=0,
$$

where $H$ now stands for the horizontal lift with respect to the Vilms connection $\omega^{C}$.

The above equation completely determines the coefficients $f^{i}$ in $\Gamma_{1}=v^{a} \tilde{E}_{a}^{C}+v^{i} X_{i}^{C}+f^{i} X_{i}^{V}+$ $0 \tilde{E}_{a}^{V}$. We may thus conclude that the primary un-reduced SODE of a couple $(\ell, \omega)$ is the unique SODE that satisfies any of the above equivalent expressions, and also $\omega^{C}\left(\Gamma_{1}\right)=X_{\omega}$ (This last property ensures that the coefficient in $\tilde{E}_{a}^{V}$ is zero. See also Proposition 4).

Remark that if $\Gamma_{1}$ satisfies this equation, then so does $\Gamma_{2}=\Gamma_{1}+V$, where $V$ is any vector field of the type $V^{a} \tilde{E}_{a}^{V}$, since

$$
\begin{aligned}
\Gamma_{2}\left(X_{i}^{V}\left(L^{H}\right)\right) & =\Gamma_{1}\left(X_{i}^{V}\left(L^{H}\right)\right)+V^{a} \tilde{E}_{a}^{V}\left(X_{i}^{V}\left(L^{H}\right)\right)=\Gamma_{1}\left(X_{i}^{V}\left(L^{H}\right)\right)+V^{a} X_{i}^{V}\left(\tilde{E}_{a}^{V}\left(L^{H}\right)\right) \\
& =\Gamma_{1}\left(X_{i}^{V}\left(L^{H}\right)\right),
\end{aligned}
$$

because $\left[\tilde{E}_{a}, X_{i}\right]=0$ and $\tilde{E}_{a}^{V}\left(L^{H}\right)=0$. In light of Proposition 6 , we conclude: 
Proposition 8. Let $\ell$ be a regular Lagrangian on $\bar{M}$ and $\bar{\Gamma}$ its corresponding SODE. Let $\omega$ be a principal connection on $\pi: M \rightarrow \bar{M}$. Any vector field $\Gamma_{2}$ on $M$ for which

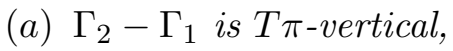

(b) $\Omega\left(\Gamma_{2}-\Gamma_{1}\right)=0$,

(c) $\Gamma_{2}$ satisfies $\Gamma_{2}\left(\left(\bar{X}^{h}\right)^{V}\left(L^{H}\right)\right)-\left(\bar{X}^{h}\right)^{C}\left(L^{H}\right)=0$, for any vector field $\bar{X}$ on $\bar{M}$,

has the property that its base integral curves project on solutions of the Euler-Lagrange equations of $\ell$.

Remark that the statement does not require the use of a specific vertical equation, as in [5]. Each choice of $\Gamma_{2}$ leads to a different vertical equation. When written in quasi-velocities, we get that $\Gamma_{2}$ is of the form $\Gamma_{2}=v^{i} X_{i}^{C}+v^{a} \tilde{E}_{a}^{C}+f^{i} X_{i}^{V}+V^{a} \tilde{E}_{a}^{V}$. As noted above, the first two terms indicate that $\Gamma_{2}$ is a SODE, and the coefficients $f^{i}$ in the third one are completely determined by the Euler-Lagrange equations of $\ell$. The only freedom left is therefore the choice of the coefficients $V^{a}$ of the fourth term, which represents the choice of a vertical equation. In the next section we discuss an example where a specific choice for that freedom is naturally available. But the advantage of our approach is the same as the one that is claimed in [5]. All the conditions can be checked on the level of the manifold $M$, and we may do so in any coordinates on $M$, not necessarily those that are adapted to $M \rightarrow \bar{M}$.

\section{Curvature distortion}

Proposition 8 showed that there are many un-reductions $\Gamma_{2}$ of the same $\bar{\Gamma}$. The best choice for $\Gamma_{2}$ may depend on the specific example one considers. In some situations, there is a natural choice. This natural choice may lead to the introduction of what was called "coupling distortion" and "curvature distortion" in [5]. We will concentrate here on the latter. To see its relation to the concepts we have introduced above, we may immediately restrict ourselves to the case of a quadratic Lagrangian, without much loss of generality.

We consider again a principal fibre bundle $M \rightarrow \bar{M}=M / G$, but now also a metric $\bar{g}$ on $\bar{M}$ and its corresponding geodesic spray $\bar{\Gamma}_{\bar{g}}$. The metric defines a quadratic Lagrangian $\ell$ on $\bar{M}$. The goal of this section is to devise a method to obtain geodesics of $\bar{g}$, without ever using coordinates on $\bar{M}$. Integral curves of $\bar{\Gamma}_{\bar{g}}$ satisfy

$$
\ddot{x}^{i}+\bar{\Gamma}_{j k}^{i} \dot{x}^{j} \dot{x}^{k}=0 .
$$

The idea of [5] is to use a reasonable construction of a metric $g$ on the un-reduced manifold $M$ and its geodesic spray $\Gamma_{g}$. That spray, however, does not have the property that its geodesics project on those of $\bar{g}$. For that reason, we need to subtract some "distortion" terms from $\Gamma_{g}$ to get a SODE $\Gamma_{2}$ which does have that property. In what follows, we wish to obtain a relation between this $\Gamma_{2}$ and our primary un-reduced SODE $\Gamma_{1}$.

The construction of the metric $g$ goes as follows. Let $B: \mathfrak{g} \times \mathfrak{g} \rightarrow \mathbb{R}$ be an Ad-invariant symmetric and non-degenerate bilinear form on $\mathfrak{g}$ (i.e., a bi-invariant metric on $G$ ). Consider again a principal connection on $\pi$. Together with $\bar{g}$ we can form a (quadratic) Lagrangian on $M$, $L=L^{H}+L^{V}$, where $L^{H}(v)=\ell(T \pi(v))=\frac{1}{2} g_{i j} v^{i} v^{j}$ and $L^{V}(v)=\frac{1}{2} B(\varpi(v), \varpi(v))=\frac{1}{2} B_{a b} v^{a} v^{b}$. The coefficients $B_{a b}$ are constants that satisfy

$$
B_{a c} C_{a c}^{d}+B_{b d} C_{a c}^{d}=0
$$

This construction of a regular Lagrangian $L$ can also be found in [6], and our notations are chosen in such a way that they match with those in [5]. 
Let us denote the (pseudo-)metric on $M$, associated to $L$, by $g$. By construction the mechanical connection of this metric is the connection we have started from, i.e., $g_{a i}=0$. The equations for the geodesics of $g$ are the so-called "Wong equations" (see again [6]). An easy way to obtain these equations goes as follows. The geodesic spray $\Gamma_{g}$ is the Euler-Lagrange SODE of $L$. As such, it is the SODE determined by

$$
\Gamma_{g}\left(X^{V}(L)\right)-X^{C}(L)=0,
$$

for any choice of vector field $X$ on $M$. If we use $X=\tilde{E}_{a}$, then we know from the invariance of the Lagrangian that $\tilde{E}_{a}^{C}(L)=0$. Therefore the corresponding Euler-Lagrange equation is

$$
\Gamma_{g}\left(B_{a b} v^{b}\right)=0
$$

meaning that the momentum $B_{a b} v^{b}$ is conserved along geodesics. This is, in essence, the "vertical" Wong equation. The "horizontal" Wong equation is the Euler-Lagrange equation we get by making use of $X=X_{i}$,

$$
\Gamma_{g}\left(X_{i}^{V}(L)\right)-X_{i}^{C}(L)=0 .
$$

Consider now again the SODE $\bar{\Gamma}_{\bar{g}}$ on $\bar{M}$ and its many un-reduced SODEs $\Gamma_{2}$, given in Proposition 8. By construction, all these vector fields satisfy

$$
\Gamma_{2}\left(X_{i}^{V}\left(L^{H}\right)\right)-X_{i}^{C}\left(L^{H}\right)=0 .
$$

Motivated by what we know about $\Gamma_{g}$, we now choose within that class, the particular SODE $\Gamma_{2}$ which has the property that

$$
\Gamma_{2}\left(B_{a b} v^{b}\right)=0 .
$$

It is easy to see that this last property uniquely determines a specific $\Gamma_{2}$ within the class of all SODEs that satisfy the conditions of Proposition 8, since it fixes the value of the coefficients $V^{a}$ in $\Gamma_{2}=v^{i} X_{i}^{C}+v^{a} \tilde{E}_{a}^{C}+f^{i} X_{i}^{V}+V^{a} \tilde{E}_{a}^{V}$. In this case, given that $B_{a b}$ is a non-degenerate constant matrix, we get simply

$$
\Gamma_{2}\left(v^{b}\right)=0,
$$

which is exactly the defining relation of the primary un-reduced SODE $\Gamma_{1}$ within the class of all $\Gamma_{2}$ 's, and thus $V^{a}=0$. Since $\Gamma_{g}$ satisfies the same equation, it has the same coefficients $V^{a}=0$, when written in quasi-velocities. The difference $A=\Gamma_{g}-\Gamma_{1}$ is therefore a vector field of the type $A=A^{i} X_{i}^{V}$. Given that $L=L^{H}+L^{V}$, and that $X_{i}^{V}\left(L^{V}\right)=0$, its coefficients $A^{i}$ are completely determined by the difference between the respective horizontal equations, i.e., by the relation

$$
A\left(X_{i}^{V}\left(L^{H}\right)\right)-X_{i}^{C}\left(L^{V}\right)=0 .
$$

If we derive the expressions of the curvature coefficients from $\left[X_{i}, X_{j}\right]=R_{i j}^{a} \tilde{E}_{a}$, then $X_{i}^{C}\left(v^{b}\right)=$ $-R_{i j}^{b} v^{j}$, we get that $X_{i}^{V}\left(L^{H}\right)=A^{j} \bar{g}_{i j}$ and $X_{i}^{C}\left(L^{V}\right)=-B_{a b} R_{i j}^{a} v^{j} v^{b}$ and thus is

$$
A^{k}=-\bar{g}^{i k} B_{a b} R_{i j}^{a} v^{b} v^{j} .
$$

The term $A$ in

$$
\Gamma_{1}=\Gamma_{g}-A
$$


is what is called "curvature distortion" in [5]. It is the term one needs to subtract from $\Gamma_{g}$ in order to get a SODE with the property that its base integral curves project on those of $\bar{\Gamma}_{\bar{g}}$. The (vertical) vector field $A$ may, of course, also be expressed in a coordinate-free manner, but we will not go into these details here.

As we saw, the effect of subtracting $A$ to $\Gamma_{g}$ is that it does not change the term of $\Gamma_{g}$ in $\tilde{E}_{a}^{V}$, but that from the term in $X_{i}^{V}$ it cancels out the curvature term in the right-hand side of the horizontal Wong equation. The point is that both $\Gamma_{g}$ and $A$, and by the above construction also $\Gamma_{1}$, can be computed in any coordinates on $M$. The above procedure is, in essence, the method that is applied in [5].

The fibration of the rotation group over the sphere. We will illustrate the discussion above by means of the realization of $\mathrm{SO}(3)$ as a $\mathbb{S}^{1}$-bundle over the sphere $\mathbb{S}^{2} \subseteq \mathbb{R}^{3}$. More precisely, for the standard metric on the sphere, we will consider the geodesics of the metric determined by $L=L^{H}+L^{V}$ and explicitly compute the "distortion" term.

We identify each rotation about the origin of $\mathbb{R}^{3}$ with an orthogonal matrix. Such a rotation $R \in \mathrm{SO}(3)$ is determined by three consecutive counterclockwise rotations, defined by the Euler angles $(\psi, \theta, \varphi)$ (following the convention of [3]):

$$
\begin{aligned}
R(\psi, \theta, \varphi) & =\left(\begin{array}{ccc}
\cos \psi & -\sin \psi & 0 \\
\sin \psi & \cos \psi & 0 \\
0 & 0 & 1
\end{array}\right)\left(\begin{array}{ccc}
1 & 0 & 0 \\
0 & \cos \theta & -\sin \theta \\
0 & \sin \theta & \cos \theta
\end{array}\right)\left(\begin{array}{ccc}
\cos \varphi & -\sin \varphi & 0 \\
\sin \varphi & \cos \varphi & 0 \\
0 & 0 & 1
\end{array}\right) . \\
& =\left(\begin{array}{ccc}
\cos \psi \cos \varphi-\cos \theta \sin \psi \sin \varphi & -\cos \psi \sin \varphi-\cos \theta \sin \psi \cos \varphi & \sin \psi \sin \theta \\
\sin \psi \cos \varphi+\cos \theta \cos \psi \sin \varphi & -\sin \psi \sin \varphi+\cos \theta \cos \psi \cos \varphi & -\cos \psi \sin \theta \\
\sin \varphi \sin \theta & \cos \varphi \sin \theta & \cos \theta
\end{array}\right) .
\end{aligned}
$$

The group $\mathbb{S}^{1}$ acts on $\mathrm{SO}(3)$ on the left by rotations about the $z$-axis, namely as:

$$
\left(\alpha \in \mathbb{S}^{1}, R(\psi, \theta, \varphi) \in \mathrm{SO}(3)\right) \mapsto\left(\begin{array}{ccc}
\cos \alpha & -\sin \alpha & 0 \\
\sin \alpha & \cos \alpha & 0 \\
0 & 0 & 1
\end{array}\right) \cdot R(\psi, \theta, \varphi) \in \mathrm{SO}(3)
$$

Note that two elements $R_{1}, R_{2} \in \mathrm{SO}(3)$ are in the same orbit if their last row, which parameterizes a sphere $\mathbb{S}^{2} \subset \mathbb{R}^{3}$, coincides. This defines a principal $\mathbb{S}^{1}$-bundle $\pi: \mathrm{SO}(3) \rightarrow \mathbb{S}^{2}$. In terms of the Euler angles the projection is $\pi(\psi, \theta, \varphi)=(\theta, \varphi)$, and the infinitesimal generator (spanning the vertical distribution) is $\tilde{E}=\partial / \partial \psi$.

In what follows, we will use the principal connection on $\pi: \mathrm{SO}(3) \rightarrow \mathbb{S}^{2}$, given by the connection form $\varpi=d \psi+\cos \theta d \varphi$. This is, in fact, the mechanical connection of the invariant Lagrangian on $T(\mathrm{SO}(3))$, given by

$$
\tilde{L}=\frac{1}{2}\left(\dot{\theta}^{2}+\dot{\varphi}^{2} \sin ^{2} \theta\right)+\frac{1}{2}(\dot{\psi}+\dot{\varphi} \cos \theta)^{2} .
$$

(For the mechanical connection, we regard $\tilde{L}$ as a metric and define the horizontal space of the connection as the space that is orthogonal to the vertical space of $\pi$.) The Lagrangian $\tilde{L}$ corresponds to a Lagrange top with equal moments of inertial, see [3]. For this principal connection, we may give the following basis of horizontal vector fields on $\mathrm{SO}(3)$ :

$$
X_{1}=\frac{\partial}{\partial \theta}, \quad X_{2}=\frac{\partial}{\partial \varphi}-\cos \theta \frac{\partial}{\partial \psi} .
$$

Quasi-velocities w.r.t. the frame $\left\{X_{1}, X_{2}, \tilde{E}\right\}$ will be denoted $\left\{v^{1}=\dot{\theta}, v^{2}=\dot{\varphi}, w=\dot{\psi}+\dot{\varphi} \cos \theta\right\}$.

Consider now the standard metric $\bar{g}=d \theta^{2}+\sin ^{2} \theta d \phi^{2}$ on $\mathbb{S}^{2}$. Its geodesic equations are given by the Euler-Lagrange equations of the Lagrangian $\ell=\dot{\theta}^{2}+\sin ^{2} \dot{\phi}^{2}$ on $T \mathbb{S}^{2}$, or by the geodesic 
spray

$$
\bar{\Gamma}_{\bar{g}}=\dot{\theta} \frac{\partial}{\partial \theta}+\dot{\varphi} \frac{\partial}{\partial \varphi}+\sin \theta \cos \theta \dot{\varphi}^{2} \frac{\partial}{\partial \dot{\theta}}-2 \cot \theta \dot{\theta} \dot{\varphi} \frac{\partial}{\partial \dot{\varphi}} .
$$

Its primary un-reduced SODE is the vector field on $T(\mathrm{SO}(3))$ given by

$$
\begin{aligned}
\Gamma_{1}=\Gamma_{\omega}+\bar{\Gamma}_{\bar{g}}^{H}= & \dot{\psi} \frac{\partial}{\partial \psi}+\dot{\theta} \frac{\partial}{\partial \theta}+\dot{\varphi} \frac{\partial}{\partial \varphi}+\sin \theta \cos \theta \dot{\varphi}^{2} \frac{\partial}{\partial \dot{\theta}}-2 \cot \theta \dot{\theta} \dot{\varphi} \frac{\partial}{\partial \dot{\varphi}} \\
& +(\sin \theta \dot{\theta} \dot{\varphi}+2 \cot \theta \cos \theta \dot{\theta} \dot{\varphi}) \frac{\partial}{\partial \dot{\psi}} .
\end{aligned}
$$

We now contrast this with the procedure of [5], where the system is un-reduced by subtracting curvature distortion from the Euler-Lagrange equations of a certain $L=L^{H}+L^{V}$. A bi-invariant metric on the Lie algebra of $\mathbb{S}^{1}$ is specified by a real number $B$. If we set $L^{V}=B w^{2}$, we may write

$$
L=\dot{\theta}^{2}+\sin ^{2} \theta \dot{\varphi}^{2}+B(\dot{\psi}+\dot{\varphi} \cos \theta)^{2},
$$

which determines a metric $g$ on $\mathrm{SO}(3)$. Its geodesic spray is

$$
\begin{aligned}
\Gamma_{g}= & \dot{\psi} \frac{\partial}{\partial \psi}+\dot{\theta} \frac{\partial}{\partial \theta}+\dot{\varphi} \frac{\partial}{\partial \varphi}+\left(\sin \theta \cos \theta \dot{\varphi}^{2}-B \sin \theta \dot{\varphi} w\right) \frac{\partial}{\partial \dot{\theta}} \\
& +\left(-2 \cot \theta \dot{\theta} \dot{\varphi}+\frac{B}{\sin \theta} \dot{\theta} w\right) \frac{\partial}{\partial \dot{\varphi}}+(\sin \theta \dot{\theta} \dot{\varphi}+2 \cot \theta \cos \theta \dot{\theta} \dot{\varphi}-B \cot \theta \dot{\theta} w) \frac{\partial}{\partial \dot{\psi}}
\end{aligned}
$$

where $w$ is short-hand for $\dot{\psi}+\dot{\varphi} \cos \theta$. Clearly, $\Gamma_{g}$ is not yet one of the un-reduced SODEs of $\bar{\Gamma}_{\bar{g}}$, and curvature distortion is required.

In the previous paragraph, we had concluded that $\Gamma_{1}$ is the unique un-reduced SODE within the class of vector fields $\Gamma_{2}$ which has the property that $\Gamma_{1}(B w)=0$. From the expressions above, it follows that the curvature distortion is given by

$$
A=\Gamma_{g}-\Gamma_{1}=-B \sin \theta \dot{\phi} w \frac{\partial}{\partial \dot{\theta}}+\frac{B \dot{\theta} w}{\sin \theta}\left(\frac{\partial}{\partial \dot{\varphi}}-\cos \theta \frac{\partial}{\partial \dot{\psi}}\right)
$$

This is clearly related to the coefficient of the curvature $\left[X_{\theta}, X_{\varphi}\right]=\sin \theta \tilde{E}$, by means of the inverse of the metric matrix $\left(\bar{g}_{i j}\right)=\left(\begin{array}{cc}1 & 0 \\ 0 & \sin ^{2} \theta\end{array}\right)$.

To end this paragraph, we sketch an alternative approach, without invoking coordinates on $\mathbb{S}^{2}$. We refer the reader to $[3,10]$ for details omitted here. The Lie algebra $\mathfrak{s o}(3)$ of $\mathrm{SO}(3)$ is given by the set of skew symmetric matrices. It has the following basis

$$
e_{1}=\left(\begin{array}{ccc}
0 & 0 & 0 \\
0 & 0 & -1 \\
0 & 1 & 0
\end{array}\right), \quad e_{2}=\left(\begin{array}{ccc}
0 & 0 & 1 \\
0 & 0 & 0 \\
-1 & 0 & 0
\end{array}\right), \quad e_{3}=\left(\begin{array}{ccc}
0 & -1 & 0 \\
1 & 0 & 0 \\
0 & 0 & 0
\end{array}\right)
$$

We will identify $\mathfrak{s o}(3)$ with the Lie algebra $\mathbb{R}^{3}$ (given by the cross product “ $\times$ "):

$$
\mathbb{R}^{3} \ni \omega=\left(\begin{array}{l}
\omega_{1} \\
\omega_{2} \\
\omega_{3}
\end{array}\right) \mapsto[\omega]=\left(\begin{array}{ccc}
0 & -\omega_{3} & \omega_{2} \\
\omega_{3} & 0 & -\omega_{1} \\
-\omega_{2} & \omega_{1} & 0
\end{array}\right)
$$

The left and right invariant vector fields corresponding to the basis vectors will be denoted by $\left(e_{i}\right)_{\ell}$ and $\left(e_{i}\right)_{r}$, and we will use $\left(e^{i}\right)_{\ell}$ and $\left(e^{i}\right)_{r}$ for the dual basis. Then, on $\mathfrak{s o}(3)$, one may 
consider the invariant inner product $\langle A, B\rangle_{\mathfrak{s o}(3)}=$ trace $\left(A B^{-1}\right)$ and its associated metric on $\mathrm{SO}(3)$, for which $\left\{\left(e^{i}\right)_{\ell}\right\}$ and $\left\{\left(e^{i}\right)_{r}\right\}$ are orthonormal moving frames. In terms of the Euler angles, this metric is the one given by the Lagrangian $\tilde{L}$ we had mentioned before.

Suppose now that $\gamma \in \mathbb{S}^{2}$. If $\pi(R)=\gamma$, then we have $\dot{\gamma}=T \pi(\dot{R})=-[\omega] \gamma=\gamma \times \omega$. The connection form is $\varpi=\left(e^{3}\right)_{r}$ and its curvature 2 -form is the area element on $\mathbb{S}^{2}$. Finally, the horizontal lift of a vector $\dot{\gamma}$ to $R$ is the element $\dot{R}$ for which $R^{-1} \dot{R}=[\dot{\gamma} \times \gamma]$, see [10]. One might also identify a rotation with an an oriented orthonormal frame $(v, w, v \times w)$ in $\mathbb{R}^{3}$ (the rotation needed to move the standard $\mathbb{R}^{3}$ basis onto the new frame). With this in mind, we pick $\hat{e}_{1}, \hat{e}_{2} \in \mathbb{R}^{3}$ such that $\left\{\hat{e}_{1}, \hat{e}_{2}, \gamma\right\}$ is orthonormal. The horizontal lift $v^{h}$ of a vector $v=\left(u_{1} \hat{e}_{1}+u_{2} \hat{e}_{2}\right)$ to an element $R \in \mathrm{SO}(3)$ is the tangent vector $\dot{R}$ such that

$$
R^{-1} \dot{R}=\left[\left(u_{1} \hat{e}_{1}+u_{2} \hat{e}_{2}\right) \times \gamma\right]=\left[u_{2} \hat{e}_{1}-u_{1} \hat{e}_{2}\right] .
$$

In other words, $v^{h}=u_{2}\left(\hat{e}_{1}\right)_{r}-u_{1}\left(\hat{e}_{2}\right)_{r}$. In this expression it is understood that $\left(\hat{e}_{1}\right)_{r},\left(\hat{e}_{2}\right)_{r}$ are evaluated at the point $R$ to which we are lifting. Therefore, one has an explicit construction of horizontal vectors in $\mathrm{SO}(3)$, and one can compute $L=L^{H}+L^{V}$ without further difficulty.

\section{Extending the un-reduction method and further outlook}

So far we have considered SODEs $\Gamma_{2}$ which have the properties that all their base integral curves project on those of $\bar{\Gamma}$. We now indicate that there may also exist SODEs $\Gamma_{3}$ which have the property that only some of their base integral curves project on those of $\bar{\Gamma}$.

We consider a principal connection $\omega$ given. We will denote its horizontal distribution by $\mathcal{H} \subset$ $T M$. Integral curves of $\bar{\Gamma}$ satisfy $\ddot{x}^{i}=f^{i}(x, \dot{x})$. Consider again the primary un-reduced SODE $\Gamma_{1}=v^{i} X_{i}^{C}+f^{i}\left(x^{j}, v^{j}\right) X_{i}^{V}+v^{a} \tilde{E}_{a}^{C}+0 \tilde{E}_{a}^{V}$. Its integral curves satisfy $\ddot{x}^{i}=f^{i}(x, \dot{x})$ and $\dot{v}^{a}=0$, together with some reconstruction equation that is primarily associated to the part $X_{\omega}=v^{a} \tilde{E}_{a}^{C}$ of $\Gamma_{1}$. Recall that we have shown in Proposition 5 that integral curves of $\Gamma_{1}$ with a horizontal initial velocity are horizontal lifts of base integral curves of $\bar{\Gamma}$ and that $\Gamma_{1}$ is tangent to $\mathcal{H}$.

Consider now a SODE of the type $\Gamma_{3}=v^{i} X_{i}^{C}+F^{i}\left(x^{j}, v^{j}, v^{a}\right) X_{i}^{V}+v^{a} \tilde{E}_{a}^{C}+F^{a}\left(x^{j}, v^{j}, v^{a}\right) \tilde{E}_{a}^{V}$, but with the property that $F^{i}\left(x^{j}, v^{j}, v^{a}=0\right)=f^{i}\left(x^{j}, v^{j}\right)$ and $F^{a}\left(x^{j}, v^{j}, v^{a}=0\right)=0$, i.e., $\left.\Gamma_{3}\right|_{\mathcal{H}}=\left.\Gamma_{1}\right|_{\mathcal{H}}$. For example, besides the property on $F^{i}$, one could simply have that $F^{a}=0$. (This property, that there is no component along $\tilde{E}_{a}^{V}$, can be characterized by saying that the SODE $\Gamma_{3}$ satisfies $\Omega\left(\Gamma_{3}\right)=\omega^{C}\left(\Gamma_{3}\right)$.) Integral curves of such a $\Gamma_{3}$ through $m_{0}$ with initial velocity $v_{0}^{i} X_{i}\left(m_{0}\right)+v_{0}^{a} \tilde{E}_{a}\left(m_{0}\right)$ satisfy, for sure, $v^{a}(t)=v_{0}^{a}$. As long as we consider integral curves of $\Gamma_{3}$ with a horizontal initial velocity, i.e., with $v_{0}^{a}=0$, they will satisfy $v^{a}(t)=0$, and $\left(x^{i}(t), v^{i}(t)\right)$ will be solutions of

$$
\ddot{x}^{i}=F^{i}\left(x^{j}, v^{j}, 0\right)=f^{i}\left(x^{j}, v^{j}\right) .
$$

With other words, some integral curves of $\Gamma_{3}$, namely the horizontal ones, will project on those of $\bar{\Gamma}$.

This situation actually also occurs in the example with Wong's equations. The quadratic spray $\Gamma_{g}$ is not one of the un-reduced SODEs of $\bar{\Gamma}_{\bar{g}}$, but it does have the property that $\left.\Gamma_{g}\right|_{\mathcal{H}}=$ $\left.\Gamma_{1}\right|_{\mathcal{H}}$, since $\left.A\right|_{\left\{v^{a}=0\right\}}=0$. We can now relate this behaviour to what is called "horizontal shooting" in $[4,7]$.

We have shown that the geodesic spray $\Gamma_{g}$ satisfies $\Gamma_{g}\left(B_{a v} v^{b}\right)=0$. This means that its integral curves (i.e., the lifted curves in $T M$ of the base integral curves), whose fibre coordinates when written in quasi-velocities are $\left(v^{i}(t), v^{a}(t)\right)$, satisfy a conservation law (of momentum-type) $B_{a v} v^{b}(t)=\mu_{a}$. Here $\mu_{a}$ are the components of an element $\mu \in \mathfrak{g}^{*}$ along the basis $E_{a}$. The specific base integral curves which happen to have "zero momentum", $\mu_{a}=0$, are exactly those whose lifted curves remain horizontal for all $t, v^{a}(t)=0$. They, therefore, coincide with the horizontal 
lifts of base integral curves. Given that we know that $\left.\Gamma_{g}\right|_{\mathcal{H}}=\left.\Gamma_{1}\right|_{\mathcal{H}}$, it follows that Proposition 5 represents a generalization of the method of "horizontal shooting" for geodesic problems.

One may find in the literature some results on what could be called "un-un-reduction". The inverse procedure of un-reduction should of course be a kind of reduction process. It is, however, not Lagrange-Poincaré reduction, since the corresponding reduced Lagrange-Poincaré equations can not necessarily be associated to a SODE on $\bar{M}$. But, for an arbitrary $G$-invariant SODE $\Gamma$ on $T M$, it makes sense to wonder whether it is $T \pi$-related to a (yet to be determined) SODE $\bar{\Gamma}$ on $\bar{M}$. An answer to this question is given in the papers on submersive SODEs. A SODE $\Gamma$ on $M$ is said to be "submersive" in $[12,16]$ if there exists a projection $\pi: M \rightarrow N$ for which it is projectable to a SODE $\bar{\Gamma}$ on $N$. In the theory of $[12,16]$ the projection $\pi$ is part of the unknowns, but if we assume that $\pi$ is a given principal fibre bundle $\pi: M \rightarrow \bar{M}$ from the outset, being submersive means that $\Gamma$ is an un-reduction of an (unknown) SODE $\bar{\Gamma}$ in $\bar{M}$. The next proposition can be found as Theorem 3.1 in [12].

Proposition 9. Let $M \rightarrow \bar{M}$ be a principal bundle and let $\Gamma$ be a SODE on $M$. Under the following two conditions $\Gamma$ is submersive:

(1) $\left[\Gamma, \tilde{\xi}^{C}\right]=0$ (with other words, it is G-invariant),

(2) $\left[\tilde{\xi}^{V}, \Gamma\right]-\tilde{\xi}^{C}$ is tangent to $\mathfrak{g}^{V}$.

Proof. If $\left\{E_{a}\right\}$ is a basis for $\mathfrak{g}$, then "being tangent to $\mathfrak{g}^{V}$ " means that the expression in (2) is of the type $A^{a} \tilde{E}_{a}^{V}$. If we write, as before, $\Gamma$ in the form

$$
\Gamma=v^{i} X_{i}^{C}+v^{a} \tilde{E}_{a}^{C}+F^{i} X_{i}^{V}+F^{a} \tilde{E}_{a}^{V}
$$

with $F^{i}, F^{a}$ functions on $T M$, then condition (2) implies that $\tilde{E}_{b}^{V}\left(F^{i}\right)=0$, while from condition (1) we get that $\tilde{E}_{b}^{C}\left(F^{i}\right)=0$ (and also that $\left.\tilde{E}_{b}^{C}\left(F^{a}\right)=C_{b c}^{a} F^{c}\right)$. Therefore, the function $F^{i}$ is $T G$-invariant, and it defines a function $f^{i}$ on $T \bar{M}$. The SODE on $\bar{M}$ given by $\bar{\Gamma}=\dot{x}^{i} \partial / \partial x^{i}+$ $f^{i} \partial / \partial \dot{x}^{i}$ is the one for which $\Gamma$ is an un-reduction.

With the results of $[12,16]$ in mind, it would be of interest to be able to "un-reduce" a SODE $\bar{\Gamma}$ on a manifold $\bar{M}$ to any bundle $\pi$ for which $\bar{M}$ happens to be the base (given a connection on that bundle), not just a principal bundle $M \rightarrow \bar{M}=M / G$. Results which may point in that direction are Theorem 1.5 in [12], or Theorem 2.1 in [16], which give conditions for a SODE $\Gamma$ on $M$ to be submersive. Moreover, some of the concepts that we have mentioned in this paper transfer to this more general context. For example, one may still define a Vilms connection on the bundle $T M \rightarrow T \bar{M}$, corresponding to a connection on an arbitrary fibre bundle $M \rightarrow \bar{M}$. However, constructing something that is similar to the vertical connection $\Omega$ (that has lead us to the definition of $X_{\omega}$ and the primary un-reduced SODE $\Gamma_{1}$ ) seems to be a more challenging task.

\section{Acknowledgements}

EGTA thanks the CONICET for financial support through a Postdoctoral Grant. TM is a visiting professor at Ghent University: he is grateful to the Department of Mathematics for its hospitality.

\section{References}

[1] Arnaudon A., Castrillón López M., Holm D.D., Covariant un-reduction for curve matching, arXiv:1508.05325.

[2] Arnaudon A., Castrillón López M., Holm D.D., Un-reduction in field theory, with applications, arXiv:1509.06919. 
[3] Arnol'd V.I., Mathematical methods of classical mechanics, Graduate Texts in Mathematics, Vol. 60, 2nd ed., Springer-Verlag, New York, 1989.

[4] Bauer M., Bruveris M., Michor P.W., Overview of the geometries of shape spaces and diffeomorphism groups, J. Math. Imaging Vision 50 (2014), 60-97, arXiv:1305.1150.

[5] Bruveris M., Ellis D.C.P., Holm D.D., Gay-Balmaz F., Un-reduction, J. Geom. Mech. 3 (2011), 363-387, arXiv:1012.0076.

[6] Cendra H., Marsden J.E., Ratiu T.S., Lagrangian reduction by stages, Mem. Amer. Math. Soc. 152 (2001), $\mathrm{x}+108$ pages.

[7] Cotter C.J., Holm D.D., Geodesic boundary value problems with symmetry, J. Geom. Mech. 2 (2010), 51-68, arXiv:0911.2205.

[8] Crampin M., Mestdag T., Routh's procedure for non-abelian symmetry groups, J. Math. Phys. 49 (2008), 032901, 28 pages, arXiv:0802.0528.

[9] de León M., Marrero J.C., Martínez E., Lagrangian submanifolds and dynamics on Lie algebroids, J. Phys. A: Math. Gen. 38 (2005), R241-R308, math.DG/0407528.

[10] Ehlers K., Koiller J., Montgomery R., Rios P.M., Nonholonomic systems via moving frames: Cartan equivalence and Chaplygin Hamiltonization, in The Breadth of Symplectic and Poisson Geometry, Progr. Math., Vol. 232, Birkhäuser Boston, Boston, MA, 2005, 75-120, math-ph/0408005.

[11] Kolář I., Michor P.W., Slovák J., Natural operations in differential geometry, Springer-Verlag, Berlin, 1993.

[12] Kossowski M., Thompson G., Submersive second order ordinary differential equations, Math. Proc. Cambridge Philos. Soc. 110 (1991), 207-224.

[13] Mestdag T., Crampin M., Invariant Lagrangians, mechanical connections and the Lagrange-Poincaré equations, J. Phys. A: Math. Theor. 41 (2008), 344015, 20 pages, arXiv:0802.0146.

[14] Nomizu K., Invariant affine connections on homogeneous spaces, Amer. J. Math. 76 (1954), 33-65.

[15] O'Neill B., Semi-Riemannian geometry: with applications to relativity, Pure and Applied Mathematics, Vol. 103, Academic Press, Inc., New York, 1983.

[16] Sarlet W., Prince G.E., Crampin M., Generalized submersiveness of second-order ordinary differential equations, J. Geom. Mech. 1 (2009), 209-221.

[17] Thompson G., Variational connections on Lie groups, Differential Geom. Appl. 18 (2003), 255-270.

[18] Vilms J., Connections on tangent bundles, J. Differential Geometry 1 (1967), 235-243.

[19] Yano K., Ishihara S., Tangent and cotangent bundles: differential geometry, Pure and Applied Mathematics, Vol. 16, Marcel Dekker, Inc., New York, 1973. 